\title{
II. ENTWICKLUNGSZUSAMMENARBEIT
}

Tomokazu Asaumi

Mehmet Günsür

Christina Kükenshöner

Oliver Lömker 


\section{Einleitung}

Seit Jahrzehnten versuchen die Industrienationen mit finanziellen und technischen Hilfsmaßnahmen die gewaltigen Disparitäten, die zwischen den OECD-Ländern und den sogenannten „Entwicklungsländern“ bestehen, abzubauen. Die Strategien, welche zum Erreichen dieses Zieles angewendet werden, sind häufig verschieden, nicht zuletzt, weil Entwicklungspolitik vielfach Interessenspolitik ist, bei der ökonomische Aspekte Vorrang vor sozialen Fragen erhalten.

Auch auf den Untersuchungsgegenstand dieser Arbeit - deutsche und japanische Entwicklungszusammenarbeit - ist diese These anzuwenden. Allerdings ist der Kern der Arbeit nicht die Kritik, sondern das Erarbeiten von Lösungsvorschlägen für eine gemeinsame Entwicklungspolitik vor dem Hintergrund einer sich verändernden Welt.

Entwicklungszusammenarbeit bedeutet nach dem allgemeinen Verständnis zunächst Geber-NehmerZusammenarbeit. Diese Form der bilateralen Beziehungen macht international den bedeutendsten Anteil der Entwicklungshilfe aus. Das zweite traditionelle Instrument staatlicher Entwicklungshilfe ist die Vergabe über multilaterale Organisationen. Beide Kooperationsformen werden in dieser Arbeit dargestellt und bewertet. Diese Betrachtung bildet die Grundlage für den Hauptteil.

Die gewonnenen Erkenntnisse werden dann genutzt, um die Kernthese der Arbeit - Deutschland und Japan sollten auf dem Gebiet der Entwicklungszusammenarbeit verstärkt kooperieren (Geberkooperation) - zu stützen, ihre möglichen Implikationen aufzuzeigen und Möglichkeiten des gemeinsamen Vorgehens darzulegen.

Kooperation setzt ein Interesse voraus, weshalb zunächst diese Frage für das deutsch-japanische Verhältnis geklärt wird. Um die Relevanz einer deutsch-japanischen Kooperation zu untermauern, wird das Thema der Entwicklungszusammenarbeit in den übergeordneten Rahmen der ,erweiterten Sicherheit“ gestellt. Aus diesem Ansatz wird auch klar, warum die möglichen Kooperationsfelder über das traditionelle Maß der Entwicklungszusammenarbeit hinausgehen und etwa den Themenkomplex „Prävention finanzieller Krisen“ mit beinhalten. Im Zeitalter der Globalisierung müssen neue Konzepte aufgegriffen werden, um auf veränderte Rahmenbedingungen angemessen reagieren zu können. Warum sollten Deutschland und Japan in der Entwicklungszusammenarbeit kooperieren? Wo liegen Gemeinsamkeiten, wo Unterschiede? Lassen sich Anknüpfungspunkte finden, die ein gemeinsames Vorgehen sinnvoll und gerechtfertigt erscheinen lassen? Wie kann eine Kooperation prinzipiell, aber auch praktisch ausgestaltet sein? Die Beantwortung dieser und weiterer Fragen führt letztendlich zu den Empfehlungen, die den Abschluß dieser Arbeit bilden.

\section{Heutige Situation der Entwicklungszusammenarbeit in Deutschland und Japan}

\subsection{Bilaterale Ebene}

\subsubsection{Ziele, Art und Verteilung der Entwicklungszusammenarbeit}

Die Entwicklungspolitik Deutschlands und Japans zeigt heute in vielen Bereichen ähnliche Schwerpunkte und Zielsetzungen. Wurde in der Vergangenheit seitens Japan Entwicklungszusammenarbeit (EZ) noch zur Sicherung von Rohstoffen aus den Entwicklungsländern genutzt und Hilfe hauptsächlich zum Aufbau einer materiellen Infrastruktur dort geleistet, so setzt die ODA-Charter (ODA - Official Development Assistance) beider Länder heute ihre Themenschwerpunkte in Bereichen der Armutsbekämpfung, des Umweltschutzes und der Bildung. Unter Achtung dreier zentraler Belange - produktives Wirtschaftswachstum, soziale Gerechtigkeit und ökologische Nachhaltigkeit - zielt EZ auf eine allgemeine Verbesserung der Lebensbedingungen der Menschen. 
Betrachtet man den Umfang der EZ, so stellt sich Japan absolut als weltgrößter ODA-Zahler dar (1996: 9,439 Mrd. US\$). Relativ zum BSP gesehen, nimmt Japan mit 0,22\% jedoch nur den 19. Rang unter den DAC-Mitgliedsländern ein (0,25\% gilt als Durchschnittswert). Deutschland positioniert sich mit 0,33\% auf Platz 10 (absolut: 1996: 7,601 Mrd. US\$).

Japan leistete traditionell einen großen Anteil seiner ODA-Zahlungen in die materielle Infrastruktur und mit 30\% (1998) läßt sich dieser Anteil immer noch als erheblich bezeichnen. Verglichen mit früheren Zahlen (1981: 45\%) ist jedoch eine Trendwende erkennbar. Entsprechend seiner ODA-Charter versucht Japan, sich stärker in Bereichen der sozialen Infrastruktur zu engagieren, welche in der deutschen ODA traditionell einen Schwerpunkt einnimmt (34,6\%).

EZ wird gemäß der DAC-Kriterien in Form von Schenkungen (dies umfaßt auch die technische Hilfe) und Krediten, die mindestens ein Zuschusselement von $25 \%$ haben müssen, geleistet. Vergleicht man das Schenkung-Kredite-Verhältnis beider Länder, so ist der Unterschied frappierend. Während auf der einen Seite die deutsche ODA mit einem Verhältnis von 90:10 als außerordentlich „sozial“ bezeichnet werden kann, wird die japanische ODA aufgrund ihres hohen Kredit-Anteils (60:40) international kritisiert. Diese Kritik sollte jedoch vorsichtig angewandt werden, da Japan stärker als Deutschland Kredite in Länder mit mittelhohem Einkommen (MIC - middle income countries) leistet, wo im Gegensatz zu den am wenigsten entwickelten Ländern (LLDC - least developed countries) Kredite durchaus ein geeignetes Anreizsystem darstellen und Schenkungen eine Gefahr im Sinne von moral hazard bedeuten können.

In Bezug auf die Liefergebundenheit der EZ ist Japan mit 99\% ungebundenen Krediten international führend. Mit dieser Politik soll auch eine Imageaufbesserung der japanischen EZ erreicht werden. Es bestehen jedoch international Zweifel an der damit demonstrierten Abkehr von wirtschaftlichen Eigeninteressen, da vermutet wird, daß auch weiterhin Aufträge an japanische Tochterunternehmen und Joint Ventures in den entsprechenden Entwicklungsländern erteilt werden. Deutschlands Kredite dagegen sind nur zu ca. $60 \%$ ungebunden.

Was die regionale Verteilung der EZ betrifft, so fällt bei Japan eine starke Konzentration auf die Region Asien auf. Japan tätigt dort knapp 50\% seiner ODA-Ausgaben, dabei insbesondere in Südostasien. Die Asienzentrierung, die seit den 80ern um 14\% zugenommen hat, wird begründet mit dem enormen Wachstumspotential Asiens und dem Mangel an für andere Regionen ausreichend qualifiziertem Personal. Im Falle Deutschlands dagegen verteilen sich die entwicklungspolitischen Ausgaben relativ gleichmäßig auf die vier vorgegebenen Großräume: 1. Sub-Saharan Afrika (31,6\%), 2. Nord Afrika und der Nahe Osten $(17,6 \%), 3$. Asien $(24,7 \%)$ und 4. Lateinamerika $(21,6 \%) .{ }^{4}$ Deutschland vertritt damit seinen „Grundsatz der globalen Strukturpolitik“.

In Bezug auf Ziele und Ausrichtung der EZ läßt sich abschließend festhalten, daß der Umweltschutz in den Entwicklungsländern als eine der zentralen Zielsetzungen der zukünftigen Entwicklungspolitik Japans gesehen wird. EZ soll weniger für die Förderung der Außenwirtschaft, sondern als Mittel zur indirekten Förderung der außenpolitischen Interessen Japans eingesetzt werden. Dies beinhaltet auch ein Umdenken zugunsten gesellschaftspolitischer Fragen wie beispielsweise der Menschenrechte. Es findet demnach ein Angleichung der Ziele und gesellschaftspolitischen Prinzipien der deutschen und japanischen Entwicklungszusammenarbeit statt.

Um einen Überblick über die relevanten Akteure in der deutschen und japanischen öffentlichen Entwicklungshilfe zu geben, wird im folgenden näher auf die Organisationsstruktur der EZ beider Länder eingegangen.

4 Der Restwert von 4,5\% bezieht sich auf Europa und wird hier nicht weiter berücksichtigt. 


\subsubsection{Organisationsstruktur der öffentlichen Entwicklungshilfegeber}

Die Organisation der öffentlichen Entwicklungshilfe in Deutschland und Japan zeigte bisher signifikante Unterschiede. Auf deutscher Seite existiert seit den 60er Jahren ein eigenes Ministerium, das Bundesministerium für wirtschaftliche Zusammenarbeit und Entwicklung (BMZ), während die japanische Entwicklungshilfe auf den ersten Blick zwar hauptsächlich in den Geschäftsbereich des Außenministeriums (Ministry of Foreign Affairs - MoFA) fällt, jedoch drei weitere Akteure maßgeblichen Einfluß auf die Entwicklungspolitik nehmen können. Beide Länder durchlaufen zur Zeit einen Organisationswechsel, allerdings sind die Auswirkungen auf japanischer Seite als wesentlich ausgeprägter zu beurteilen, während die interministeriellen Kompetenzverschiebungen in Folge des Regierungswechsels in der Bundesrepublik nur geringe Veränderungen bewirken werden.

Die deutsche Entwicklungshilfe ist geprägt durch ein pluralistisches System. Die Definition der entwicklungspolitischen Rahmenbedingungen und der sich daraus ergebenden tatsächlichen Projekte, Hilfen etc. erfolgt vornehmlich durch das BMZ. Die Implementierung wird schwerpunktmäßig von zwei thematisch voneinander getrennten Organisationen, der Kreditanstalt für Wiederaufbau (KfW) und der Deutschen Gesellschaft für technische Zusammenarbeit (GTZ) vorgenommen. Das Auswärtige Amt (AA) nimmt unter den übrigen bundesdeutschen Ministerien, welche je nach Schwerpunkt eines Projektes hinzugezogen werden können, eine besondere Rolle ein. Entwicklungspolitik kann in starkem Maße auch auswärtige Politik sein, immanent ist sie es zumindest fast immer. Über die deutschen Botschaften wird in den Empfängerländern die EZ koordiniert und repräsentiert.

Weitere Akteure und Organisationen deutscher Entwicklungspolitik sind bundeseigene Gesellschaften, halbstaatliche Organisationen, freie Träger, sowie einige Bundesländer und Kommunen.

Wie bereits oben angedeutet ist das japanische Außenministerium nur auf den ersten Blick der entscheidende Akteur japanischer Entwicklungspolitik. Die Organisation japanischer EZ im Geflecht der Ministerien zeigt, daß es einige weitere sehr entscheidende Akteure gibt. Hierbei sind vor allem zu nennen: das Ministry of Financial Affairs (MoF), das Ministry of International Trade and Industry (MITI) und die Economic Planning Agency (EPA).

Bilaterale staatliche Entwicklungshilfe in Form von Schenkungen (grant aid) und technischer Hilfe wurde und wird auch in Zukunft in erster Linie vom MoFA initiiert. Die Implementierung dieser Hilfe erfolgt größtenteils über die Japan International Cooperation Agency (JICA). JICA hat den Status einer Körperschaft des öffentlichen Rechts, untersteht aber der Aufsicht des MoFA und kann daher keine eigenständige Politik betreiben. Allerdings soll JICA zusätzliche Kompetenzen erhalten, um das MoFA für Strategiebildung und Koordination zu entlasten. Der Overseas Economic Cooperation Fund (OECF), der unter der Aufsicht des MoFA, des MoF, des MITI und der EPA zinsgünstige Kredite zur Projektfinanzierung (yen loans) vergibt, soll ebenfalls verstärkt entlastend für das MoFA dienen. Diese Veränderungen erfolgen im Zuge der Reform der japanischen Administration, bei der voraussichtlich bis zum Jahr 2000 die Anzahl der Ministerien von 22 auf 12 reduziert wird. Dies wird auch Auswirkungen auf die japanischen ODA-Programme haben. Nach dem heutigen Stand der Reform der Ministerien werden die drei entwicklungspolitischen „Konkurrenten“ des MoFA auch weiterhin ein Mitspracherecht behalten, jedoch wird die Rolle des MoFA gestärkt, da es de jure der Koordinator der japanischen ODA-Aktivitäten wird. Ein weiterer Akteur japanischer Entwicklungshilfe, der gleichwohl nach den Kriterien der OECD nicht zur ODA zu rechnen wäre, ist die Japan Export-Import Bank (JEXIM), deren Kredite kommerziellen japanischen Interessen dienen und die der gleichen Aufsicht wie des OECF unterliegt. Hier ist eine womöglich entscheidende Veränderung beschlossen worden. OECF und JEXIM sollen bis Oktober 1999 verschmelzen und personell gemeinsam arbeiten. Die Verbindung einer nach kommerziellen Gesichtspunkten agierenden Bank mit einer Durchführungsorganisation der ODA wirft natürlich die Frage nach Integritätsverlusten auf. Dem soll durch getrennte, und im Falle der ODA, transparentere Mittelverteilung entgegengewirkt werden.

Als weitere Akteure japanischer Entwicklungshilfe wären noch zu nennen: die Außenhandelsorganisation JETRO, sowie das ihr angegliederte Institute for Developing Economies (IDE). Sie unterstehen dem MITI. 
Auch die Japan Development Bank (JDB) leistet Entwicklungshilfe im Sinne von technischer Unterstützung bei der Vermittlung von Know-how für Länder, die sich im Übergang zu einem marktwirtschaftlichen System befinden. Neben den genannten vier Ministerien können auch die anderen Ministerien Japans ähnlich wie im Falle der Bundesrepublik - bei konkreten Projekten der Entwicklungshilfe ihre Expertise zur Verfügung stellen. Nicht-Regierungsorganisationen spielen in der japanischen Politik eine eher untergeordnete Rolle. Dies liegt zum Teil an dem nicht geklärten Rechtsstatus solcher Organisationen in Japan. Allerdings gibt es in den letzten Jahren vereinzelte Ansätze in der japanischen Entwicklungspolitik, dieses zu ändern. So hat das MoFA 1994 eine „NGO Assistance Division“ geschaffen.

Beide Länder zeigen also gewisse Gemeinsamkeiten, aber vor allem auch starke Unterschiede in der Organisationsstruktur der staatlichen Entwicklungshilfe. Die deutsche Variante eines eigenen EZMinisteriums hat sicherlich einige Vorteile gegenüber dem bisherigen japanischen System. Allerdings spiegelt die japanische Version auch deutlich den realpolitischen Ansatz japanischer EZ wider. Japanische EZ ist in wesentlich stärkerem Maße ein offensichtliches Mittel der Außen- und der Außenwirtschaftspolitik, sowie der außenpolitischen Darstellung des Landes. Inwieweit durch die beschlossenen Strukturänderungen der japanischen Entwicklungspolitik tatsächlich zu mehr Effizienz und Transparenz verhelfen werden kann, ist momentan nicht differenziert zu beurteilen. Allerdings läßt die angestrebte Kompetenzkonzentration die Vermutung zu, daß die Ziele leichter erreicht werden könnten.

Trotz der unterschiedlichen Organisationsstrukturen laufen Projekte der bilateralen staatlichen Entwicklungszusammenarbeit in beiden Ländern im Prinzip gleich ab. Variationen ergeben sich besonders im Moment noch in einzelnen Projektphasen durch die dargelegten, andersgelagerten Kompetenzverteilungen.

\subsection{Multilaterale Ebene}

\subsubsection{Position Deutschlands und Japans zur multilateralen Entwicklungszusammenarbeit}

Unter multilateraler EZ werden all jene Beiträge verstanden, die nicht aufgrund bilateraler Vereinbarungen direkt an ein Entwicklungsland gehen, sondern über multilaterale Entwicklungsbanken (MDB) und sonstige internationale Organisationen geleistet werden. Vorteile weist der Multilateralismus in der EZ gegenüber dem Bilateralismus insofern auf, als das Finanzmittel und auch qualifiziertes Personal in einem größeren Umfang zur Verfügung stehen, so daß Projekte und Programme durchgeführt werden können, die für ein einzelnes Land nicht zu realisieren wären. Auch läßt sich durch multilaterale EZ dem Vorwurf des Eigennutzes entgehen, der ja auch Japan in starkem Maße angelastet wurde (Rohstoffsicherung, Exportförderung der eigenen Industrie). Dennoch ist derzeit ein Trend weg vom Multilateralismus feststellbar, der auch auf Japan und Deutschland zutrifft. Die folgende Abbildung 1 veranschaulicht die Entwicklung der Zahlungen an multilaterale Organisationen seit 1990. 
Abb.1: Zahlungen an multilaterale Organisationen im Verhältnis zum BIP

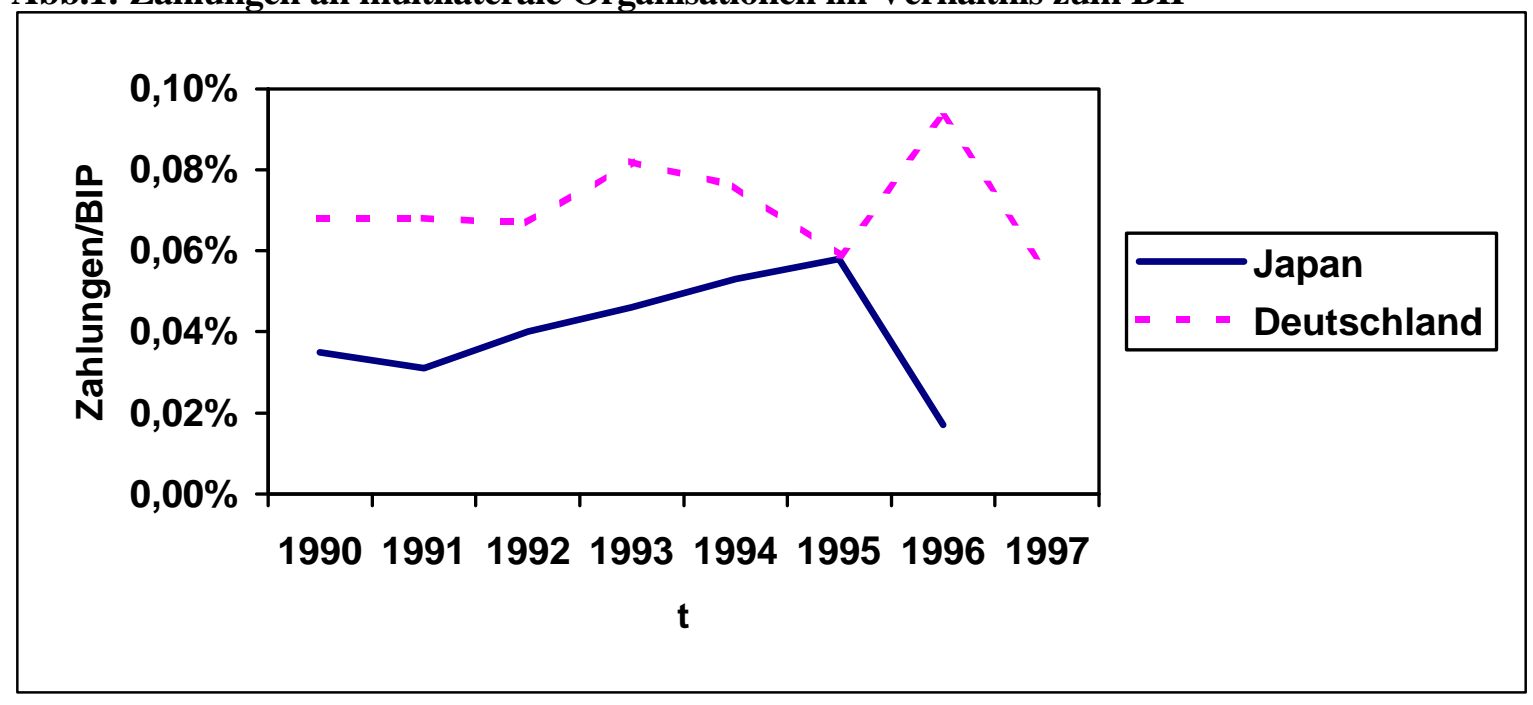

Quelle: MoFA (1998): S. 48, BMZ: Statistiken und Berichtswesen, in: Internet: http://www.bmz.de/epolitik/statistiken/stat-05a.pdf und eigene Darstellung.

In Japan erfolgte 1998 eine über 10\%ige Kürzung des ODA-Budgets, die zum großen Teil auf die schwere Haushaltskrise zurückgeführt werden kann. Innerhalb dieser Einsparung sollte die multilaterale Hilfe, die viele Jahre als Markenzeichen der japanischen EZ galt, mit ca. $40 \%$ zuerst überproportional stark reduziert werden. Dies konnte nach interministeriellen Machtkämpfen durch das MoFA abgewendet werden, dennoch wurden die Leistungen an die Entwicklungsbanken, für die das MoF verantwortlich zeichnet, um fast 20\% gekürzt. Gegenwärtig werden ca. $16 \%$ der japanischen ODA an multilaterale Organisationen gezahlt. Gründe für Japans Abkehr vom Multilateralismus lassen sich zum einen in dem Bestreben finden, entsprechend internationalen Forderungen qualitativ bessere EZ zu leisten, anstatt nur auf Quantität zu setzen und daraus abgeleitet eine Konzentration auf bilaterale Hilfe. Zum anderen spielt auch der Umstand eine wichtige Rolle, daß die außenpolitischen Ziele, die mit den hohen Zahlungen an internationale Organisationen verfolgt wurden, nicht erreicht werden konnten. Statt einer Stärkung der Stellung in internationalen Organisationen zog sich Japan vielmehr das Image der „Scheckbuchdiplomatie“ zu.

Auch in Deutschland ist eine - wenn auch keine plötzliche - Abkehr vom Multilateralismus erkennbar. Etwa 1/3 des ODA-Haushaltes wird noch an multilaterale Organisationen geleistet. Hiervon werden jedoch $60 \%$ an die EU gezahlt, so daß über einen großen Teil der multilateralen Hilfe nicht mehr von deutscher Seite über die Mittelverwendung bestimmt wird.

Was die EU anbetrifft, so ist der Haushalt der gemeinschaftlichen ODA zweigeteilt. Über den Europäischen Entwicklungsfond (EEF), der sich aus nationalen Beiträgen der Mitglieder finanziert, werden mit rund 45\% des EZ-Gesamthaushalts 71 Länder Afrikas, der Karibik und des Pazifiks (AKP) im Rahmen der Konvention von Lomé unterstützt. Hilfe an Länder Asiens, Lateinamerikas, des Mittelmeerraums, Mittel- und Osteuropas sowie die Neuen Unabhängigen Staaten der ehemaligen Sowjetunion wird aus dem Haushalt der EU finanziert. Ihr Anteil liegt folglich bei 55\% des EZGesamthaushalts. Die Mittel, welche die Bundesrepublik für die Entwicklungshilfe der EU aufbringt, werden nicht über das Budget des BMZ verwaltet, sondern in diesem besonderen Fall gibt es eine gemeinsame Verantwortlichkeit von BMZ, AA, Finanzministerium und Wirtschaftsministerium. Die neue Bundesregierung hat allerdings in ihrem Koalitionsvertrag vereinbart, daß das BMZ die Federführung in Fragen der EU-Entwicklungspolitik übernehmen soll.

Auch wenn dem BMZ zufolge das politische und finanzielle Hauptgewicht auf der bilateralen EZ liegt, gibt es seit dem Regierungswechsel Ende letzten Jahres Bemühungen, die Ausgaben für multilaterale EZ zu erhöhen. Nach Aussagen der Bundesministerin für wirtschaftliche Zusammenarbeit und Entwicklung, Heidemarie Wieczorek-Zeul wolle man sich zukünftig in internationalen Organisationen, wie Weltbank, 
Internationaler Währungsfonds und UN-Sonderorganisationen wieder mehr engagieren. Ein Grundstein dafür sei durch die 11\%ige Aufstockung der Zahlungen an die IDA (International Development Agency), d.h. 2,2 Mrd. DM für drei Jahre, gelegt worden.

In den folgenden beiden Abschnitten wird auf Deutschlands und Japans Position innerhalb von multilateralen Organisationen eingegangen: in UN-Sonderorganisationen und - als Beispiel einer Regionalbank - in der Asiatischen Entwicklungsbank.

\subsubsection{Stellung Deutschlands und Japans in UN-Sonderorganisationen}

Japan ist mit einem Anteil von 17,98\% nach den USA (25\%) der zweitgrößte Beitragszahler an die Vereinten Nationen gefolgt von Deutschland mit 9,63\%.

Trotz hoher Beitragszahlungen sind Deutschland und besonders Japan in der UN personell unterrepräsentiert. Zwar kann sich der Personalanteil nicht nur am Beitragsanteil ausrichten, da sonst die Entwicklungsländer stark benachteiligt werden würden, dennoch sind Japan und Deutschland im Vergleich zu Ländern, die finanziell einen geringeren Anteil besitzen, personell unterbesetzt. Um dieser Tatsache entgegenzuwirken und den Anteil deutschen Personals in UN-Sonderorganisation zu erhöhen, gibt es von deutscher Seite aus die Nachwuchsförderung des BMZ „Beigeordnete Sachverständige“, sowie das von der Zentralstelle für Arbeitsvermittlung in Frankfurt (ZAV) ins Leben gerufene „Büro Führungskräfte zu internationalen Organisationen (BFIO)“, welches Jobs und Praktikumsstellen in internationalen Organisationen vermittelt. Um einen kurzen Überblick über die Stellung Deutschlands und Japans in den wichtigsten UN-Sonderorganisationen zu geben, sind deren Beiträge in Tabelle 1 zusammengefaßt.

Tab.1: Beiträge Deutschlands und Japans zu UN-Sonderorganisationen (1997)

\begin{tabular}{|l|l|l|l|l|}
\hline $\begin{array}{l}\text { UN-Sonder- } \\
\text { organisationen }\end{array}$ & \multicolumn{2}{l}{ Deutschland } & \multicolumn{2}{l|}{ Japan } \\
\hline & $\begin{array}{l}\text { Beiträge in Mio. } \\
\text { DM }\end{array}$ & Anteil(\%) & $\begin{array}{l}\text { Beiträge in Mio. } \\
\text { US\$ }\end{array}$ & Anteil(\%) \\
\hline FAO & 28,9 & 6,5 & 53,6 & 8,8 \\
\hline IMF & $8,2^{\mathrm{a}}$ & $5,7^{\mathrm{b}}$ & $8,2^{\mathrm{a}}$ & $5,7 \mathrm{~b}$ \\
\hline UNDP & 120,0 & 26,8 & 99,3 & 16,3 \\
\hline UNESCO & 16,8 & 3,8 & 57,1 & 9,4 \\
\hline UNEP & 9,6 & 2,1 & 6,0 & 1,0 \\
\hline UNFPA & 42 & 9,4 & 54,4 & 8,9 \\
\hline UNHCR & 8,9 & 2,0 & 115,7 & 19,0 \\
\hline UNICEF & 9,9 & 2,2 & 28,0 & 4,6 \\
\hline UNRWA & 10,3 & 2,3 & 19,9 & 3,3 \\
\hline WFP & 45,0 & 10,1 & 99,0 & 16,2 \\
\hline WHO & 50,6 & 11,3 & 63,2 & 10,4 \\
\hline
\end{tabular}

Quelle: MoFA: ODA Annual Report 98, S. 164; BMZ: Statistik und Berichtswesen, in Internet: http://www.bmz.de/epolitik/statistiken/stat-05a.pdf und eigene Darstellung.

Anmerkungen: a in Mrd. SZR

b Anteil an der Gesamtquote zum IMF

Einige UN-Sonderorganisationen werden zur Zeit auch von Deutschen oder Japanern geleitet. So wird das UNEP (United Nations Environment Programme) von Klaus Töpfer und das UNHCR (United Nations High Commissioner for Refugees) von Sadako Ogata geleitet. 


\subsubsection{Die Asiatische Entwicklungsbank}

Innerhalb des multilateralen Sektors leisten auch die Regionalen Entwicklungsbanken, in die Deutschland ca. 3\% seiner ODA einzahlt, einen Beitrag zur Entwicklungshilfe. Unter diesen soll hier die Asiatische Entwicklungsbank (Asian Development Bank - ADB) näher betrachtet werden.

Die ADB wurde 1966 in Manila gegründet und unterstützt mit Krediten, technischer Hilfe und anderen Dienstleistungen ihre Mitgliedsentwicklungsländer. Angesichts der Finanzkrisen in Asien engagiert sich die ADB auch bezüglich finanzieller Unterstützung und Durchführung institutioneller Reformen in den entsprechenden Ländern. Es wurde ein Währungsfonds (Asian Currency Crisis Support Facility, ACCSF) in Höhe von 3 Milliarden Dollar bereitgestellt, der im Fall von zukünftigen Turbulenzen auf den Kapitalmärkten Soforthilfe leisten soll.

Die Mitgliedsstaaten teilen sich auf in 41 regionale und 16 nicht-regionale Staaten. Der Entschluß, auch nicht-regionale Mitglieder zuzulassen, ist begründet in der Mobilisierung von Kapital und der Abhängigkeit von Industriestaaten als Mittelgeber.

Die Finanzmittel der ADB bestehen in sogenannten ordentlichen Finanzmitteln, aus denen Kredite zu marktähnlichen Konditionen gewährt werden und in Sonderfonds, mit denen Kredite an LLDC's gezahlt werden, die definitionsgemäß zur Entwicklungshilfe gehören (,soft loan window“). Als bedeutendster Sonderfond ist der Asiatische Entwicklungsfond (ADF) zu nennen, gegründet 1973, dessen größter Einzahler Japan ist.

Was Japan und Deutschland innerhalb der ADB anbetrifft, gehören beide Länder zu den 31 Gründungsmitgliedern, ihre Position stellt sich jedoch sehr unterschiedlich dar.

Japan entwarf bereits seit den 50er Jahren Pläne für eine asiatische Regionalbank, und diese Impulse wurden in das Konzept der ADB eingearbeitet. ${ }^{5}$ Seit Bestehen der ADB ist Japan eng mit dem Management verknüpft und stellt bisher alle Präsidenten. Dadurch kann ein großer Einfluß auf die operative Tagespolitik und die Personalpolitik ausgeübt werden. Anteilsmäßig teilt sich Japan die Position des größten Shareholders $(16,054 \%)$ mit den USA. Die Stimmrechtsverteilung basiert auf den Kapitalzeichnungen zu den ordentlichen Finanzmitteln, die bei Japan und USA dementsprechend gleich hoch sind. In die Sonderfonds zahlt Japan jedoch das ungefähr dreifache seines ADB-Anteils ein (Japan hat hier einen Anteil von über 50\%), so daß im Vergleich zu den USA insgesamt ein sehr viel höherer Beitrag von Japan geleistet wird. Ebenso wird oben erwähnter Währungsfonds ACCSF allein von Japan finanziert. Aufgrund der Führungsrolle Japans wird die ADB in der Literatur auch karikierend als „Japan Development Bank“ dargestellt.

Deutschland dagegen nimmt eine weitaus unauffälligere Position innerhalb der ADB ein. Mit einem Anteil von 4,45\% ist die Bundesrepublik zwar nach den USA und Kanada der drittgrößte Shareholder unter den nicht-regionalen Mitgliedsländern und innerhalb Europas der größte, dennoch ist der Einfluß auf die Politik der ADB eher gering. Dies wird auch durch eine Unterrepräsentanz in der personellen Besetzung deutlich.

Als Gründe für die Mitwirkung Deutschlands innerhalb der ADB sind die anerkannte entwicklungspolitische Effizienz der ADB, die Möglichkeit, Projekte durchzuführen, die aus politischen und finanziellen Gründen für eine bilaterale Kooperation nicht geeignet sind und die zusätzlichen Liefermöglichkeiten für die deutsche Wirtschaft zu nennen.

5 Die für die Durchführung der Gründung zuständige ECAFE (United Nations Economic Commission for Asia and the Far East, jetzt ESCAP) wurde unterstützt von einem Expertenteam dem zahlreiche Japaner angehörten, darunter auch der jetzige ADB-Präsident Tadao Chino (laut ADB-Pressemitteilung Nr. 70/98) 
Die Lieferaufträge der Empfängerländer werden über das sog. International Competitive Bidding ${ }^{6}$ verteilt, womit eine Bevorzugung einzelner Länder verhindert werden soll. Deshalb ist anzunehmen, daß auch Deutschland durch seine hohen Beitragszahlungen keine Vorteile in Bezug auf Lieferaufträge erringen kann. Über möglicherweise verfolgte andere Eigeninteressen läßt sich durch die Literatur wie auch durch die ADB selbst nichts erfahren. Es bleibt jedoch festzuhalten, daß angesichts der relativ hohen Beitragszahlungen ein Potential zum Ausbau des eigenen Einflusses besteht, welches durchaus für ein stärkeres Asienengagement genutzt werden könnte.

\subsection{Bisherige Kooperation zwischen Deutschland und Japan}

Schon derzeitig finden Bemühungen zur Kooperation zwischen Japan und Deutschland bei der Entwicklungszusammenarbeit statt, und zwar sowohl auf technischer wie auf finanzieller Ebene.

Im Rahmen der jährlichen Japan/ EU-Konsultationen zur Entwicklungszusammenarbeit vereinbarten Deutschland und Japan 1996 bilateral eine Koordination ihrer EZ, um eine effizientere Gestaltung der Projekte zu erreichen. Diese Koordination bezieht sich hauptsächlich auf die TZ und wird zur Zeit in 11 Ländern durchgeführt (Ägypten, Kenia, Tansania, Sambia, Zentralafrika, Bangladesch, Usbekistan, Kasachstan und Brasilien). In 5 Ländern (Sambia, Tansania, Kenia, Malawi, Simbabwe) finden auch lokal Koordinierungen von EZ statt. Das heißt, zwischen den beiden ausführenden Institutionen, JICA und GTZ, wird bezüglich ähnlicher Projektvorhaben gemeinsam vor inländischen Ministerien vorgesprochen, und es findet ein Informationsaustausch statt. Zu einer gemeinsamen Projektdurchführung ist es bis jetzt jedoch noch nicht gekommen, und vor allem von seiten der GTZ bestehen diesbezüglich Zweifel an der Realisierbarkeit. Als Grund wird dafür die Kompliziertheit der Verfahrensweisen zur Projektgenehmigung / -durch-führung auf japanischer Seite angeführt. Auch würde Japan immer noch einen anderen Ansatz zur Entwicklungshilfe verfolgen als Deutschland, der mehr von einer hardware-Politik bestimmt würde. ${ }^{7}$

Eine engere Beziehung zwischen JICA und GTZ wird durch einen Mitarbeiteraustausch gefördert, der auch Teil der Vereinbarung von 1996 war. Seither ist nur ein Mitarbeiter der JICA für ein Jahr in der GTZ gewesen, um Know-how bzgl. Funktionen und Organisationen der Institutionen auszutauschen, Verbindungen zu schaffen und spezifische Felder der gemeinsamen EZ zu koordinieren. Von der GTZ ist bisher kein Mitarbeiter bei der JICA gewesen

Darüber hinaus besteht eine Kooperation der KfW mit der JDB. Innerhalb des Programms zur Förderung von Entwicklungsbanken durch die KfW, besteht seit 1996 eine Vereinbarung, bei der technischen Unterstützung, die im Rahmen der Finanzierungshilfe zum Aufbau von Entwicklungsbanken angeboten wird, mit der JDB zusammenzuarbeiten. Umgesetzt wurde dies durch gemeinsam veranstaltete Seminare z.B. für die ungarische, kroatische und chinesische Entwicklungsbank. Bereits seit 1987 finden regelmäßige Treffen zwischen Experten der KfW und der JDB zum Zwecke des Meinungsaustausches statt. Was die finanzielle Zusammenarbeit anbetrifft, so hat die KfW bereits seit Anfang der 80er gemeinsame Kofinanzierungsprojekte mit dem OECF.

Wenn man die bisherige Zusammenarbeit zwischen Deutschland und Japan an konkreten Projekten messen wollte, so ist diese vor allem auf technischer Ebene als eher erfolglos zu bezeichnen. Dennoch läßt sich das, was bisher an Kooperation zwischen Deutschland und Japan passiert ist, als positiver Vorsatz für zukünftige Kooperationen werten. Dort, wo Entwicklungshilfe ausgeführt wird, also auf der

6 Verfahren unter Aufsicht der ADB nach dem das EL nach Zubilligung eines ADB-Kredits den zu erteilenden Auftrag international öffentlich ausschreibt und nach von der ADB festgelegten Kriterien den günstigsten Anbieter auswählt.

7 Informationen beruhen auf einem Telefonat mit dem ehemaligen Leiter des GTZ-Büros in Nairobi am 17.4.1999 
Mitarbeiterebene, sind erste persönliche Kontakte geknüpft und bieten so eine gute Basis für eine konkretere Zusammenarbeit.

\section{Möglichkeiten zukünftiger deutsch-japanischer Kooperation in der Entwicklungszusammenarbeit}

\subsection{Bewertung der Kooperationsinteressen Deutschlands und Japans}

Bevor im weiteren Verlauf der Arbeit geklärt werden kann, „wie“ Japan und Deutschland in entwicklungspolitischen Fragen kooperieren könnten, soll zunächst untersucht werden, „warum“ Kooperation für beide Seiten sinnvoll sein könnte.

Ganz simpel läßt sich sagen: Deutschland und Japan kooperieren. Sie tun dies nicht erst seit neuestem, sondern seit Jahren und dies in vielfältiger Art und Weise.

Diese Tatsache kann nicht verwundern. Sie ist Ausdruck eines historischen Prozesses, der sich in ähnlicher Weise zwischen nahezu allen Akteuren der internationalen Beziehungen, wobei hiermit an dieser Stelle explizit Nationalstaaten gemeint sind, abgespielt hat und täglich aufs neue abspielt. Ein qualitativer Unterschied in den Beziehungen zwischen Staaten ist allerdings dann erreicht, wenn Kooperation über das $\mathrm{Ma} ß$ der gegenseitigen Anerkennung hinausreicht und diplomatische Beziehungen nicht nur Ausdruck internationaler Normen des Umgangs sind. Zusammenarbeit geht im Sinne dieser Arbeit weit über die angedeuteten Kriterien hinaus. Sie bedeutet ständige Absprache, die Bereitschaft zu Kompromissen, Ehrlichkeit im Umgang, ein institutionalisiertes oder normiertes Verhalten - kurz etwas, daß ein partnerschaftliches Verhältnis skizzieren kann.

Auch diese erweiterten Kriterien treffen auf das deutsch-japanische Verhältnis zu. In einer interdependenten Welt müssen die Beziehungen zwischen zwei gleichentwickelten Industrienationen beinahe zwangsläufig intensiv sein. Allerdings ist der Ansatz zur Zusammenarbeit, der in dieser Arbeit entwickelt werden soll, letztlich doch anders zu beurteilen als etwaige Verträge über Handelsbeziehungen. Es geht um ein gemeinsames Handeln, welches dann aber zuerst begründet werden muß, ergo die Frage des ,warum“.

Die Welt, in der wir leben, hat sich verändert. Die Globalisierung führt zu Veränderungen, die teilweise erst in ihren Ansätzen zu erkennen sind, nichtsdestotrotz aber bereits heute althergebrachte Lösungsstrategien nationaler und internationaler Probleme obsolet erscheinen lassen. Die Asienkrise hat gezeigt, daß die internationalen Finanzmärkte der staatlichen Kontrolle vielfach entzogen sind. Dennoch bleibt festzuhalten, daß bei allen Autoritätsverlusten der Akteur Staat noch immer eine, wenn nicht sogar die dominante Rolle in den internationalen Beziehungen spielt. Jedoch ist einzelstaatliches Handeln schwieriger geworden. Im Falle Deutschlands wird dieser Effekt durch die europäische Integration und die damit verbundenen, gewollten Autoritätsübertragungen an die supra-staatliche EU sogar noch intensiviert.

Deutschland und Japan haben mit zum Teil unterschiedlichen Strategien und Zielen Entwicklungshilfe betrieben, dies haben die vorangegangenen Kapitel gezeigt. Gleichwohl gibt es aber auch Gemeinsamkeiten in der Entwicklungshilfe der beiden Länder. Der entscheidende Ansatz dieser Arbeit ist, daß nicht nur die Übereinstimmungen, sondern gerade auch die Abweichungen eine Chance zu sinnvoller und intensivierter Kooperation darstellen können. Da die hier aufzustellenden Strategien sich an die deutsche Außenpolitik richten, ist besonders der Blickwinkel der Verwirklichung bzw. des „Anschubes“ durch deutsche Stellen zu betrachten.

Deutsche Entwicklungszusammenarbeit ist ihrem Selbstverständnis nach „globale Strukturpolitik“. Die am Beginn dieses Kapitels skizzierten Rahmenbedingungen staatlichen Handelns haben aber die Schwierigkeiten einzelstaaatlichen Handelns gerade in einer globalisierten Welt aufgezeigt. Will Deutschland also seinen entwicklungspolitischen Anspruch verwirklichen, dann wird es in hohem Maße von 
internationaler Kooperation abhängig sein. Dies muß nicht zwangsläufig über Multilateralismus geschehen, auch das Verhältnis zwischen Geber- und Nehmerland ist ja durchaus schon als internationale Kooperation zu begreifen. Der neue Ansatz dieser Arbeit versucht aber einen dritten möglichen Weg aufzuzeigen, den des Trilateralismus in der Entwicklungszusammenarbeit.

Lassen wir bei der Betrachtung zunächst den „Dritten“, sprich das spätere Empfängerland, außen vor und widmen uns den zu untersuchenden Hauptakteuren. Dabei ist die Reduktion auf zunächst zwei Akteure zu verstehen als bilaterale (Geber-) Kooperation mit dem Ziel der trilateralen Zusammenarbeit.

Kooperation kann nur dann sinnvoll funktionieren, wenn beide Seiten dazu bereit sind und wenn es für beide Gewinne in irgendeiner Form gibt. Das prinzipielle Interesse Deutschlands und Japans an Kooperation im allgemeinen ist unverkennbar vorhanden und aus den oben erwähnten Gründen auch logisch und berechtigt. Daraus allerdings ein zwangsläufiges Interesse zur Kooperation auf dem Sektor der Entwicklungszusammenarbeit abzuleiten, wäre sicherlich eine zu vereinfachende Sichtweise der reellen Situation. Vielmehr läßt sich aus den bisherigen Politiken der beiden Länder in den meisten Fällen ein „entweder-oder"-Verhalten ableiten. Entweder bilateral oder multilateral. Wenn diese Strategien aus dem einen oder anderen Grund nicht mehr die gewünschte Wirkung erfüllen, warum dann nicht neue Wege beschreiten und über eine andere Form gemeinsamen Handelns zum Ziel kommen?

Für die entwicklungspolitische Kooperation lassen sich mehrere Gründe anführen. Multilaterale Hilfe ist in der Beliebtheitsskala der Regierungen gesunken. In Deutschland wird unter der neuen Regierung eine Trendwende versucht, die jedoch angesichts leerer Haushaltskassen nur mit hohem Aufwand und Kürzungen an anderen Stellen zu erreichen sein wird. Hinzu kommen die relativ gebundenen Gelder, die Deutschland für die EU-Entwicklungspolitik aufwenden muß, und die ja wie bereits erwähnt einen großen Teil deutscher multilateraler Hilfe ausmachen. In Japan konnte die radikale Abkehr vom Multilateralismus durch das MoFA gerade noch verhindert werden, aber auch dort zeigen sich Risse im vorher so heilen Bild des großen Gebers. Japan setzt verstärkt auf die Kenntlichmachung der gegebenen Hilfe. Wie gesagt, japanische Entwicklungshilfe ist ein Instrument der Außenpolitik. Also könnten gemeinsame Strategien wahrscheinlich am ehesten bei „bilateraler“ Hilfe greifen. Dieser Ansatz kann durch ein weiteres Argument gestützt werden - durch das Argument der Qualität. Deutsche Entwicklungshilfe hat international einen besseren Ruf als japanische. Japanische EZ aber besitzt einen finanziellen Umfang, der den deutschen um ein Vielfaches übertrifft. Eine Verbindung dieser Gegensätze könnte Synergieeffekte ergeben, die dem gemeinsamen, übergeordneten Prinzip der „Entwicklung“ neue Impulse geben können.

Auch für die deutsch-japanischen Beziehungen kann solch eine Vorgehensweise positive Effekte erzeugen. Kooperation kann die beiden Länder enger aneinander binden als dies heute der Fall ist. Somit würden bisherige Bemühungen - etwa in Form des Dialogforums - unterstützt und vertieft.

Die japanische EZ ist in erster Linie staatlich bestimmt und organisiert. Zivilgesellschaftliche Akteure spielen eine allenfalls marginale Rolle. Auch hier könnten Impulse von Deutschland an Japan ausgehen, wobei jedoch nicht übersehen werden darf, daß NGOs nicht von „oben“ verordnet werden können, sondern nur Anreize für ihr Engagement erfolgen können. Allerdings ist zumindest ein verstärkter Austausch deutscher und japanischer Erfahrungen möglich.

Erfahrungsaustausch ist auch in weiteren Feldern denkbar und hier sogar besonders wünschenswert. Beide Länder benutzen Länderanalysen für Konzepterstellungen. Ein intensiver Austausch dieser Bewertungen und Schlußfolgerungen kann beiden Seiten zur Überprüfung der Ergebnisse und zur Planung des weiteren Vorgehens dienen.

Sollte Japan bereit sein, die bisherige Praxis der Asienzentrierung aufzugeben, dann kann sich für Deutschland die Chance ergeben, seine Expertise in bezug auf andere Regionen zur Verfügung zu stellen, um somit auch die eigenen Zielen unterstützt zu sehen. Gleichzeitig kann die Bundesrepublik von den japanischen Erfahrungen, besonders aber auch seinen Kontakte in Asien profitieren. 
Für beide kann die Möglichkeit bestehen, international eine „Vorreiterrolle“ bei neuen Vorgehensweisen der EZ einzunehmen. Gerade hier kann ein Anknüpfungspunkt an die japanische Seite bestehen, da damit auch dem japanische Verständnis von Außenpolitik entgegengekommen wäre.

Schließlich sollte auch die multilaterale Ebene nicht aus den Augen verloren werden. Falls sich Deutschland und Japan auf gemeinsame Standpunkte und Strategien einigen können, dann wäre es wesentlich stärker als bisher möglich, den eigenen Einfluß in solchen Organisationen zu erhöhen, um eine eigenständige Politik zu betreiben.

Diese prinzipiellen Überlegungen sollen in den folgenden Kapiteln konkretisiert werden bzw. auf ihre Verwendbarkeit in ausgesuchten Feldern der Entwicklungspolitik untersucht werden. Um grundlegende Vorbehalte frühzeitig zu entkräften, sollen zunächst aber einige Punkte, die gegen Kooperationen sprechen könnten, problematisiert und entkräftet werden.

Erstens: auch bei gemeinsamem Vorgehen müßten nicht allzu teure und ressourcenverschlingende Organisationsformen entstehen. Der Punkt also, der häufig und in gewisser Weise auch zu Recht gegen multilaterale Organisationen vorgebracht wird, kann nach Meinung der Autoren vermieden werden. Vorhandene Strukturen müssen nach neu aufzustellenden Regeln genutzt werden bzw. vorhandene Ansätze müssen intensiviert werden. Zweitens: der differierende Aufbau deutscher und japanischer EZ in bezug auf die ministerielle Kompetenzverteilung scheint sich abzubauen. Werden mehr Kompetenzen als bisher an das MoFA delegiert, dann bietet sich gerade hier für die deutsche Seite der Versuch an, Kooperation anzustreben. Der Ansprechpartner in Japan ist wohlbekannt und gegenseitige Konsultationen, aber auch schon tiefgreifendere Partnerschaften etwa im Bereich der Botschaftszusammenarbeit sind vorhanden und könnten für gemeinsame EZ-Programme genutzt werden.

\subsection{Entwicklungspolitische Kooperation im Zeichen einer 'erweiterten Sicherheitspolitik'}

Interesse und Anreize zu einer Kooperation im Bereich der Entwicklungszusammenarbeit bestehen also sowohl bei Deutschland als auch bei Japan, wie oben gezeigt wurde.

Wo soll nun die gemeinsame Kooperation ansetzen? In vielen unterschiedlichen Gebieten der Entwicklungspolitik sind gemeinsame Projekte denkbar, die sich allein aufgrund der möglichen Synergienbildung für eine Kooperation anbieten würden. Es ist jedoch zu befürchten, daß bei bloß einzelnen, unabhängigen gemeinsamen Aktionen die Tragweite der Bedeutung einer gemeinsamen Kooperation verloren geht. Daher ist es sinnvoll, den Kooperationsbestrebungen als Ganzes einen Rahmen zu geben, ein umfassendes Konzept, durch die den gemeinsamen Bemühungen - auch international - eine tiefere Bedeutung zukommen könnte. Ein Rahmen, der diesen Ansprüchen gerecht werden kann und vielerlei Möglichkeiten zur Kooperation bietet, ist der der ,erweiterten Sicherheitspolitik‘.

Der Begriff der Sicherheitspolitik an sich läßt sich eher im militärischen Bereich vermuten, doch in seinem erweiterten Verständnis kommt der Entwicklungspolitik eine bedeutende Rolle zu. Für die Sicherheit der Menschen ist ein friedliches Zusammenleben eine Grundbedingung, die nur unter bestimmten sozialen wie auch ökonomischen Voraussetzungen gewährleistet werden kann. Auf diese kann die Entwicklungspolitik Einfluß üben, indem sie langfristige Fragen der Krisenursachen und der gesellschaftlichen Bedingungen des Zusammenlebens bearbeitet. In der Agenda für den Frieden 1992 werden beispielsweise Armut, Bevölkerungswachstum und Umweltzerstörung als die zentralen weltweiten Sicherheitsrisiken genannt. Doch auch ökonomische Probleme haben eine sicherheitspolitische Dimension. So hat die asiatische Wirtschaftskrise gezeigt, daß durch die politischen und sozialen Folgen die Sicherheit der betroffenen Länder bedroht wurde, aber auch global gesehen, die Auswirkungen auf die internationalen Finanzsysteme erheblich sind. Zusammengefaßt läßt sich sagen, daß Entwicklungszusammenarbeit im Sinne einer erweiterten Sicherheitspolitik an einer Verbesserung der wirtschaftlichen, sozialen, ökologischen und politischen Verhältnissen in anderen Weltregionen arbeitet. 
,Erweiterte Sicherheitspolitik' ist ein Rahmen, in den Arbeitsbereiche wie z.B. Armutsbekämpfung oder auch Ausbildung, eingefaßt werden können, in denen beide Länder bereits aktiv sind. In Deutschland ist das Thema der erweiterten Sicherheitspolitik gerade sehr aktuell - davon zeugt nicht zuletzt die Diskussion um einen zivilen Friedensdienst und der Sitz des BMZ im Bundessicherheitsrat - und daher auch interessant für einen weiteren Ausbau im Hinblick auf eine Kooperation. Auch in Japan ist der Gedanke der erweiterten Sicherheitspolitik nicht neu. Unter dem Begriff der „,comprehensive securities“ (sôgô anzen hoshô) war er vor allem in den 80er Jahren in der Diskussion. Für Japan müßte es interessant sein, diesen Ansatz auch auf die EZ auszuweiten, da er eine Plattform bietet, um sich in Bereichen (z.B. der der sozialen Infrastruktur) zu profilieren, die dem in letzter Zeit stärker verfolgten software-Ansatz der EZ entsprechen (vgl. Kapitel 2.1).

Darüber hinaus ist dieser Themenkomplex geeignet, um auch international Anerkennung zu gewinnen. Auch vor dem Hintergrund der Zurückhaltung bei PKO-Einsätzen in der Vergangenheit, mußten sich gerade Deutschland und Japan den Vorwurf der „Scheckbuchdiplomatie“ gefallen lassen. Hier anzusetzen und - anstatt auf Krisen zu reagieren - diesen im Sinne einer erweiterten Sicherheitspolitik präventiv vorzugreifen, müßte für beide Länder ein sehr interessanter Ansatz sein. Daß dies langfristig eine bessere Position innerhalb von UN-Organisationen bewirken könnte, ist durchaus vorstellbar.

Um sowohl der gesellschaftlichen wie auch der ökonomischen Komponente gerecht zu werden, soll im folgenden in zwei gedankliche Abschnitte unterteilt, vorgegangen werden. Welche Kooperationsmöglichkeiten im Zeichen der ,erweiterten Sicherheit' sind denkbar im gesellschaftlichen Bereich, d.h. bei der Krisenprävention und -reaktion gesellschaftlicher Konflikte und welche sind denkbar im ökonomischen Bereich, explizit der Finanzkrise in Asien?

\subsubsection{Krisenprävention/ -reaktion gesellschaftlicher Konflikte}

\section{Ursachen und Reaktionen}

Demokratie sowie politische und gesellschaftliche Stabilität sind durchaus nicht global abgedeckte, in sämtlichen Nationen gewährleistete Phänomene. In unterentwickelteren Staaten ist der Alltag häufig gezeichnet durch Armut, Analphabetentum, Umweltkatastrophen, Menschenrechtsverletzungen und wirtschaftliche Rückständigkeit, die unter Umständen Krisen aller Art verursachen. Finanzstarke Industrienationen, sprich Geberländer der Entwicklungspolitik, setzen sich mit Spenden oder Krediten das Ziel, eine grundlegende Stabilität in diesen potentiellen Krisenregionen aufzubauen, bzw. Krisenursachen an der Wurzel zu bekämpfen. Problematisch sind Krisen, da sie die menschliche Entwicklung und damit das allgemeine Ziel der Entwicklungszusammenarbeit behindern, nämlich gemeinsam die globale Entwicklung voranzutreiben. Im schlimmsten Fall eskalieren Krisen hin zu kriegerischen oder bürgerkriegsähnlichen Auseinandersetzungen. Solche Konflikte entstehen durch ungleichgewichtige Verteilungen, beispielsweise zwischen Volk und politischer Elite, zwischen Arm und Reich und aus ethnischen Gründen bzw. durch eine allgemeine Unzufriedenheit des Volkes. Die Entwicklungszusammenarbeit soll nun helfen, diese Konfliktpotentiale abzubauen und kann daher als krisenpräventive Funktion dargestellt werden. Im folgenden geht es hauptsächlich darum, Möglichkeiten für eine Förderung friedlicher Konfliktlösungen darzulegen, wobei in der Argumentation den NGOs (NonGovernmental Organizations) eine besondere Rolle zukommt. Konflikte sind heutzutage in zunehmendem Maße innerstaatliche Konflikte. Die Konfliktbearbeitung solcher Krisen ist im System der Vereinten Nationen wegen des Prinzips der Nichteinmischung besonders schwierig. Deshalb können NGOs - wenn auch nicht ausschließlich, so doch ergänzend - ein geeignetes Instrument darstellen, da sie zivilgesellschaftliche Akteure darstellen.

Deutschland und Japan versuchen mit großem Aufwand, soziale Disparitäten zu verringern. Eine in den letzten Jahren verstärkt genutzte Möglichkeit ist die Einbindung der NGOs in die staatlichen, entwicklungspolitischen Konzeptionen bzw. das Bemühen, Kooperations- und Koordinationsmöglichkeiten mit NGOs zu verbessern. Die NGOs gelten als sehr wichtige Partner im Bereich der „software aid“, 
insbesondere der Nothilfe, Bildung, Umwelt und Wasserversorgung, also der Gebiete, die besonders im Hinblick auf die Bekämpfung von Krisenursachen auf dem Programm stehen. Deshalb ist es überlegenswert, die komparativen Vorteile der NGOs verstärkt den staatlichen Hilfsorganisationen zu eigen zu machen. Diese Vorteile zeichnen sie aus in ihren Basiszielsetzungen, ihrer Flexibilität und in ihren häufig besseren Kenntnissen der lokalen bzw. regionalen Bedürfnisse. NGOs sind beispielsweise in Deutschland in Form von kirchlichen Organisationen, Vereinen oder anderen privaten Institutionen vorhanden. Über 500 deutsche Nicht-Regierungsorganisationen werden zum Teil großzügig vom BMZ finanziell unterstützt (ca. 10\% seines Budgets, ca. 7\% der gesamten ODA-Mittel werden für NGOAktivitäten aufgewendet). Die Zahl der weltweit existierenden Nicht-Regierungsorganisationen beläuft sich auf eine fünfstellige Zahl, wobei vor allem die Frage der „NGO-Definition“ großen Spielraum in der Zählweise zuläßt. In Japan zeigen sich bei den NGOs starke Organisationsmängel. Das NGO-Netzwerk ist auch im DAC-Vergleich sehr unterentwickelt und nicht besonders stark ausgeprägt. Jedoch bezieht sich die Unterentwicklung nicht nur auf die Anzahl, der in Japan existenten NGOs. 1994 wurden 276 solcher Organisationen gezählt. Sie sind jedoch charakterisiert durch ihre geringe Ausstattung im Budgetumfang (80\% Budgetvolumen < 1 Mio. US\$), womit der Personalmangel begründet wird. Außerdem ist diese Art der Organisation recht jung (60\% wurden nach 1984 gegründet). Anteilsmäßig liegt der Umfang der NGOAktivitäten geringer als 3\% der japanischen ODA-Programme. Es sind in letzter Zeit jedoch viele Versuche gestartet worden, mit mehr Engagement und Förderung der NGOs die Effektivität der Hilfsprogramme im allgemeinen zu steigern und zunächst kleine ODA-Hilfen auf bestimmte Gebiete zu konzentrieren.

Vielfach werden jedoch Versuche, die für mehr unabhängiges Handeln oder unkontrollierteren Handlungsspielraum der NGOs in Japan plädieren, strikt von der Ministerialbürokratie abgelehnt. Finanzielle Mittel für NGOs werden überwiegend vom japanischen Außenministerium zur Verfügung gestellt; zwei über das MoFA Hauptquartier (1. „non-profit foundation subsidy”, 2. „,subsidy system for NGO projects in developing countries”) und ein Instrument aus lokalen „MoFA-Missions” (3. ,grand assistence for grassroots projects").

Die NGO-Szene übt auch zunehmend Kritik an der ODA-Politik. Die Vorwürfe sind nicht neu, sondern greifen die bekannten Argumente auf, nach denen Japans ODA überwiegend für kommerzielle Interessen instrumentalisiert wird, auf Umweltbelange und Menschenrechtsverletzungen $\mathrm{zu}$ wenig Rücksicht genommen wird und die Orientierungskraft der „ODA-Charta” im Vollzug der ODA zu gering erscheint.

In ihrem Kern ist die Aufgabe der Krisenprävention keine neue „Sache”. Es wird jedoch verstärkt das Ziel verfolgt, zur Konfliktvermeidung jeglicher Art gezielt Entwicklungszusammenarbeit einzusetzen und die bisherigen Tätigkeiten der Entwicklungspolitik zu ergänzen. Auch das BMZ sieht sich vor der Aufgabe, mit Instrumenten der Entwicklungszusammenarbeit, Krisenpotentiale in einem frühen Stadium zu erkennen und zu analysieren, und den Aspekt der Krisenprävention mit Hilfe von Länderberichten, -konzepten und studien zu erweitern. Sowohl in Deutschland als auch in Japan wird versucht - wenngleich gerade in Japan noch starke Widerstände spürbar sind - eine engere Zusammenarbeit zwischen NGOs und staatlichen Stellen, sowie eine Koordinierung der NGOs untereinander anzustreben. Hier sind also aufgrund dieser ähnlichen Entwicklung Kooperationsmöglichkeiten gegeben.

Der bedeutendste Unterschied zwischen der deutschen und japanischen NGO-Szene liegt in der finanziellen Ausstattung und ihrer Akzeptanz. Anders als in Deutschland, wo seit Dezember 1995 eine Dachorganisation deutscher NGOs (VENRO) auch einen großen Einfluß auf staatliche entwicklungspolitische Institutionen und Entscheidungen ausüben kann, sind dagegen die japanischen NGOs deutlich unterrepräsentiert und haben weniger Expertise. 


\section{Mögliche Ebenen der Kooperation}

Das Gesamtbild zeigt, daß Deutschland und das etwas „hinterherhinkende” Japan in eine gleiche Richtung abzielen. Es ist aber momentan noch schwierig, auf NGO-Ebene Kooperationsmöglichkeiten zu realisieren. Dafür müßten die NGOs in Japan die Möglichkeit haben, kompetenter aufzutreten. Trotzdem kann auf staatlicher Ebene durchaus eine Kooperation möglich sein. Ganz allgemein kann man folgende vier Möglichkeiten von Kooperationsformen, welche in den Empfehlungen auch wieder aufgegriffen werden, formulieren:

1) ,ad-hoc Koalition”: diese Form der Zusammenarbeit könnte sich beispielsweise für Nothilfemaßnahmen, insbesondere bei Folgen von Naturkatastrophen als vorteilhaft erweisen

2) „formalisiertes Vorgehen”: eine gegenseitige Absprache zwischen Deutschland und Japan zur Entwicklung von gewissen Formen der Kooperation oder Vorgehensweisen bei einzelnen Projekten, beispielsweise wenn es um die Bekämpfung von Krisenursachen oder Förderung friedlicher Konfliktlösungen geht.

3) Kooperation auf institutioneller Ebene: das Einrichten neuer gemeinsamer Institutionen, die gezielt das Thema der Krisenprävention auf die Tagesordnung setzen und neue Projekte schmieden.

4) „,informelles Vorgehen”: gegenseitiger Austausch von Informationen und Erfahrungen, als ein Element zur effizienteren und produktiveren Zusammenarbeit bei gemeinsamen Projektgestaltungen.

Bereits laufende Projekte Deutschlands und Japans, die auf Krisenursachenbekämpfung, Förderung friedlicher Konfliktlösungen und Nothilfemaßnahmen abzielen, zeigen nicht selten Parallelen, insbesondere in Teilbereichen wie der Wasserversorgung, Grundbildung und Nahrungsmittelversorung. Durch Kooperationen könnten unnötige Kosten gespart werden und eine höhere Produktivität erzielt werden, beispielsweise in Form einer ,regionalen Ergänzung”. Japan ist besonders in Asien aktiv, wie bereits im ersten Teil dieser Arbeit deutlich gemacht wurde, ist aber auch im afrikanischen Raum mit einer deutlich geringeren Zahl an Projekten vertreten, wo wiederum einer von Deutschlands Schwerpunkten liegt. Durch eine enge Zusammenarbeit in Form der oben genannten vier Möglichkeiten, die keineswegs nur als alternativ zu betrachten sind, könnten erhebliche Vorteile erzielt werden.

\subsubsection{Krisenprävention/ -reaktion finanzieller Krisen am Beispiel Asien}

Wie bereits einleitend in Kapitel 3.2 erwähnt, spielt auch der ökonomische Aspekt eine wichtige Rolle bei dem Begriff der erweiterten Sicherheitspolitik. Daher soll in diesem Kapitel auf mögliche Kooperationen im Bereich der Prävention bzw. Bewältigung finanzieller Krisen eingegangen werden. Aus Gründen der Aktualität wird hier eine Eingrenzung auf die Asienkrise vorgenommen. Der Kürze wegen wird im folgenden der Begriff der „Asienkrise“ verwendet. Es muß jedoch angemerkt werden, daß „die Asienkrise“ ein vereinfachender Begriff von mehreren Krisen in der Region Asien ist.

Deutschland als exportorientierte Nation hatte durch die Asienkrise einen starken Rückgang der Exporte in asiatische Länder zu verzeichnen (Bsp. Thailand: 1. Hälfte 1998 ggü. 1.Hälfte 1997: -34\%). Auch aufgrund hoher Kredite deutscher Banken in Asien mußten wegen der Krise Verluste hingenommen werden. Allein dies sollte für Deutschland genug Motivation darstellen, sich bei der Bewältigung der Krise mehr als durch Zahlungen an den IMF zu engagieren. Japan mit seiner Präsenz in Asien kann dabei einen interessanter Kooperationspartner darstellen. Bevor jedoch konkret auf Vorschläge zur Kooperation eingegangen wird, sollen kurz die Ursachen und Folgen der Asienkrise und anschließend bereits ergriffene Hilfsmaßnahmen und deren Kritik erläutert werden. 


\section{Ursachen und Folgen der Asienkrise}

Die asiatische Finanzkrise kann nicht auf eine Ursache zurückgeführt werden, denn viele verschiedene Gründe trugen zu ihr bei bzw. verschärften sie. Aus Platzgründen sollen diese hier nur stichwortartig abgehandelt werden:

1) Aufwertung der asiatischen Währungen aufgrund der Kopplung an den US\$, der seit 1995 aufgewertet wurde: Verlust der Wettbewerbsfähigkeit.

2) hohes Leistungsbilanzdefizit der asiatischen Staaten.

3) übermäßige Aufnahme von kurzfristigen Krediten aus dem Ausland ohne Risikoabsicherung (moral hazard).

4) Spekulationen auf eine Abwertung der asiatischen Währungen. Dadurch war die feste Wechselkursanbindung zum US\$ nicht mehr aufrechtzuerhalten: Abwertung der asiatischen Währungen und dadurch dramatischer Anstieg der Schuldenlast.

Die Folgen der Krise (Arbeitslosigkeit, Armut und politische Unruhen etc.) sind keineswegs auf Asien beschränkt, sondern global spürbar. Exportnationen, wie die Bundesrepublik, haben große Einbußen auf dem asiatischen Absatzmarkt zu verzeichnen. Auch ausländische Investoren, die sich in Asien betätigten, haben hohe Verluste zu vermelden. Besonders Japan steht nun vor der Aufgabe der Bewältigung einer großen Anzahl von Problemkrediten. ${ }^{8}$

\section{Geleistete Maßnahmen zur Krisenbewältigung und Lösungsvorschläge}

In Tabelle 2 werden kurz die Hilfsmaßnahmen der verschiedenen Institutionen in Reaktion auf die Finanzkrise umrissen.

Tab. 2: Von IMF, Weltbank und ADB geleistete Maßnahmen in Reaktion auf die Asienkrise

\begin{tabular}{|l|l|}
\hline IMF & $\begin{array}{l}\text { Kredite, die Reformmaßnahmen im institutionellen und makroökonomischen Bereich der } \\
\text { Programmländer (z.B. Außenhandelsliberalisierung, Umstrukturierung des Finanz- und öffentlichen } \\
\text { Sektors, geldpolitische Maßnahmen) zur Bedingung haben } \\
\text { technische Hilfe zur Umsetzung der geforderten Reformen }\end{array}$ \\
\hline Weltbank & $\begin{array}{l}\text { Kredite und technische Hilfe zur Durchführung der vom IMF geforderten Reformen } \\
\text { Kredite gegen die sozialen Auswirkungen der Krise }\end{array}$ \\
\hline ADB & $\begin{array}{l}\text { Kredite zur Unterstützung der Strukturreformen } \\
\text { Kredite zur Begrenzung der sozialen Auswirkungen } \\
\text { Etablierung eines „Regional Economic Monitoring Unit“ zur Überwachung des Banken- und } \\
\text { Finanzsektor der ASEAN-Länder }\end{array}$ \\
\hline
\end{tabular}

Quelle: IMF (1998), Weltbank (1999), ADB (1999) und eigene Darstellung.

Auch von seiten Japans wurden umfangreiche finanzielle Mittel zur Krisenbewältigung zur Verfügung gestellt. Rund 85 Mrd. US\$ stellte Japan im Rahmen der sogenannten „Miyazawa Initiative“ zur Überwindung der Asienkrise bereit. Davon wurden Zahlungen durch den IMF, aber auch bilateral in die Region vor allem für den Privatsektor und zur Importfinanzierung getätigt. Ein weiterer großer Anteil (15 Mrd. US\$) steht den asiatischen Ländern auf Abruf bei Devisenengpässen bereit. Erklärbar wird dieses hohe finanzielle Engagement vor dem Hintergrund folgenden Zusammenhangs: Einerseits trug Japan mit seiner eigenen ökonomischen Schwäche implizit zur Krise bei, indem es weniger asiatische Importe abnahm und es ist andererseits selbst stark von den Auswirkungen der Krise betroffen. Zu begründen ist dies mit

8 Detaillierte Informationen zur Asienkrise können aus der Homepage von N.Roubini entnommen werden: http://equitiy.stern.nyu.edu/ nroubini/asia/AsiaHomepage.html. 
Japans Position als Hauptkreditgeber und der Tatsache, daß 40\% des Außenhandels mit Asien geführt wird.

Mit Einsetzen der Reformmaßnahmen des IMF wurde Kritik an demselben laut. Das Festhalten an einem Standardlösungskonzept, das Kreditzusagen an eine strenge Geld- und Fiskalpolitik knüpft, sei nicht adäquat für eine Region in der die makroökonomischen Fundamentaldaten im normalen Bereich lagen, und die strengen Konditionen hätten die Krise somit sogar noch verschärft. Zum Teil auch aus dieser Kritik heraus entstanden alternative Lösungsvorschläge, von denen drei hier kurz vorgestellt werden sollen.

1) AMF (Asian Monetary Fund): anknüpfend an das Argument der Undifferenziertheit, mit der der IMF seinen Programmländern mit der Standardforderung nach einer strengen Geld- und Fiskalpolitik gegenüberstehe, wurde 1997 die Idee eines regionalen Fonds von asiatischer und vor allem von japanischer Seite angeregt. Der asiatische Währungsfonds sollte seine Ausschüttungen nicht an Strukturreformen knüpfen, sondern in erster Linie, an die Situation der Region angepaßt, stabilisierend wirken. Von amerikanischer Seite wurde der AMF jedoch heftig kritisiert und daher wurde dieser Vorschlag fallengelassen.

2) Tobin-Steuer: abhebend auf die Problematik der Spekulationen, welche ja zumindest ein auslösendes Moment der Krise waren, beinhaltet die Idee der Tobin-Steuer, daß auf grenzüberschreitende Finanztransaktionen eine Transfersteuer erhoben wird, um so den spekulativen Kapitalverkehr einzudämmen. Die Realisierbarkeit dieses Konzeptes - wer könnte es international durchsetzen - bleibt jedoch fraglich.

3) Das chilenische Modell: eingehend auf die Problematik kurzfristiger Investitionen müssen ausländische Investoren nach diesem Modell ihre Anlagegelder auf ein Jahr zinslos deponieren, wodurch kurzfristige Investitionen unprofitabel werden. Hier läßt sich kritisch anmerken, daß solche restriktiven Maßnahmen einer Deregulierung der Finanzmärkte zuwiderlaufen.

Angesichts eines sinkendes US Dollars und der Kosovokrise in Europa investieren die Anleger wegen fehlender Alternativmöglichkeiten wieder vermehrt in asiatische Länder. Daher ist die Frage wohl berechtigt, ob der Reformdruck nicht bereits nachläßt und somit auch die Bereitschaft der betroffenen Länder zu umfassenden Strukturreformen. Trotzdem möchten wir an dieser Stelle einige mögliche Ansätze der gemeinsamen Finanzkrisenintervention und -prävention Deutschlands mit Japan im finanziellen und im technischen Bereich diskutieren.

\section{Möglichkeiten der Kooperation im Bereich finanzieller Zusammenarbeit}

Um auf finanzieller Ebene effektiv einen Beitrag zur Bewältigung der Asienkrise zu leisten, erscheint die Wahl eines multilateralen Rahmens sinnvoll. Die Wirksamkeit einer rein bilateralen Geberkooperation muß im Hinblick auf das Volumen, das sie finanziell leisten kann, angezweifelt werden. Eine verstärkte Zusammenarbeit Japans und Deutschlands innerhalb von multilateralen Organisationen bietet jedoch vielversprechende Möglichkeiten, einen wirksamen Beitrag zu leisten. Rein praktische Gesichtspunkte, wie die mögliche Nutzung einer bereits vorhandenen Infrastruktur, spielen eine Rolle. Aber auch im Hinblick auf die Auswirkungen, die solch ein Engagement für die Position Deutschlands innerhalb der Organisation hat, ist eine Kooperation dieser Art für Deutschland interessant. Im folgenden sollen zwei Ansätze dazu vorgestellt werden.

Für die Nutzung einer multilateralen Organisation als Forum einer verstärkten Zusammenarbeit bietet sich aufgrund des regionalen Bezuges die ADB an. Denkbar wäre hier die Einrichtung eines gemeinsamen Fonds von Deutschland und Japan. Dieser Fonds kann, entsprechend den Statuten der ADB sich mehr für eine langfristige Entwicklung einzusetzen, beispielsweise für die Bekämpfung der sozialen Auswirkungen der Finanzkrise genutzt werden. Auch eine Bereitstellung für Währungsstabilisierungen ist denkbar, jedoch schlechter mit einem entwicklungspolitischen Ansatz zu vereinbaren. Es ist vorstellbar, daß bei einer Bereitstellung eines Fonds durch zwei Länder, die Projekte, die an eine Kreditvergabe geknüpft sind, nicht 
durch das International Competitive Bidding, sondern paritätisch an Japan und Deutschland vergeben werden, so daß der Fonds auch aus wirtschaftlichen Aspekten interessant wäre.

Interessant ist solch ein Fonds für Deutschland, abgesehen von dem entwicklungspolitischen Beitrag zur Krisenbewältigung, aber auch vor dem Hintergrund der jetzigen Position Deutschlands innerhalb der ADB. Wie in Kapitel 2.2.3 bereits näher erläutert wurde, ist diese, trotz relativ hoher Beitragszahlungen, politisch eher unbedeutend und ohne große Einflußnahme. Ein Fonds bietet die Möglichkeit einer größeren Präsenz und damit verbunden auch einer stärkeren Mitbestimmung in der ADB. Eine personelle Aufstockung ist dafür jedoch ratsam.

Darüber hinaus sind die Signale, die von solch einem offensichtlichen Engagement für Asien an die asiatischen Länder ausgehen - im Vergleich zu den Zahlungen durch den IMF, die von den Empfängerländern als „amerikanische Zahlungen“ aufgenommen werden dürften - von Vorteil für die Gewinnung von Geschäftskontakten.

Auf die Frage, warum bei solch einem Fonds eine Kooperation gerade mit Japan sinnvoll wäre, sind Gründe zu nennen, wie Japans offensichtliche Bereitschaft für die Krisenbewältigung finanziell aktiv zu werden, seine Vorreiterrolle innerhalb der ADB und die Tatsache, daß Deutschland kaum bereit sein dürfte, einen Fonds in einem ,wirksamen“ Umfang allein zu finanzieren.

Japan, auf der anderen Seite nimmt bereits eine einflußreiche Position innerhalb der ADB ein und trägt mit einem eigens finanzierten Währungsfonds in der ADB zur Krisenbewältigung bei. Dennoch wäre auch für Japan eine Kooperation mit Deutschland im Hinblick auf einen gemeinsamen Fonds attraktiv. Noch auf dem letzten Jahrestag der ADB, Anfang Mai, mußte sich Japan den Vorwürfen der USA aussetzen, innerhalb der ADB einen ,asiatischen Ansatz“ der Entwicklungspolitik zu verfolgen. Diese Aussage an sich kann auf ihren Wahrheitsgehalt hin kritisch hinterfragt werden, Fakt jedoch ist, daß die USA dem starken Einfluß Japans eher negativ gegenübersteht und die ADB in einen globalen Kontext einbinden möchte. Hier setzt die mögliche Argumentation für Deutschland als Kooperationspartner ein. Als Verfechter einer - wie es auch im Koalitionsvertrag festgeschrieben ist - ,globalen Strukturpolitik“kann Deutschland sozusagen als Garant für eben diese dienen und Japan in einen „westlichen“ Kontext einbinden. Damit ließe sich auch das Argument entkräften, daß Deutschland sich mit einem „Zusammenschluß“ mit Japan implizit gegen die USA wenden würde, denn über diesen Verdacht müßte Deutschland mit seiner eindeutigen Positionierung für die USA und den IMF erhaben sein.

Eine andere Alternative der Kooperation mit Japan im Hinblick auf finanzielle Zusammenarbeit im weiteren Sinne, liegt in der Wiederaufnahme der Idee des bereits erwähnten AMF, jedoch in einer anderen Form, als in der er bei Entstehung der Idee diskutiert wurde. Ein Fonds kann nämlich nicht nur finanzielle Soforthilfe leisten, sondern auch technische Unterstützung bieten. Letzteres ist interessant im Hinblick auf die Asienkrise und unterstützenswert von deutscher Seite, wie im folgenden gezeigt werden soll.

Als 1997 vor allem von japanischer Seite Anstoß zum AMF gegeben wurde und dieser dann am Widerstand der USA scheiterte, war er wegen seiner finanziellen Soforthilfe, die er leisten sollte, in der Diskussion. Das heißt, aufbauend auf den hohen Ersparnissen der Region Asien, sollte er seine Mittel schneller als der IMF und vor allem der wirtschaftlichen Situation Asiens entsprechender, also nicht geknüpft an Strukturanpassungen, den Krisenländern zur Verfügung stellen. In dieser Funktion hätte er eine direkte Konkurrenz für den IMF bedeutet. Im Hinblick auf die Problematik des moral hazard ist dies kein unterstützenswertes Projekt für Deutschland und auch aufgrund Deutschlands offiziellem Bekenntnis zum IMF nicht vertretbar.

Doch wie oben bereits angedeutet, können durch einen AMF auch Funktionen im Bereich der technischen Unterstützung wahrgenommen werden, die eine sinnvolle und notwendige Ergänzung zum IMF bedeuten würden. Und unter der Voraussetzung der Inanspruchnahme solcher Funktionen ist der AMF sehr wohl der Unterstützung Deutschlands wert. 
In Ergänzung zu den Artikel 4-Konsultationen" ${ }^{9}$ des IMF kann der AMF als eine Art Frühwarnsystem dienen. Aufgrund des möglichen Übergreifens (contagion) einer Krise auf das eigene Land, liegt es im eigenen Interesse der asiatischen Länder, Probleme, die zu solch einer Krise führen können, zu identifizieren. Der AMF könnte als Institution dienen, in der solche Probleme evaluiert werden, um dann Druck auf die Regierungen ausüben zu können, so daß auf erkannte Probleme Reaktionen erfolgen müssen. Es ist anzunehmen, daß ein AMF eine höhere Akzeptanz innerhalb Asiens und damit verbunden auch mehr Durchsetzungskraft erreichen kann als der IMF.

Vor diesem Hintergrund ist der AMF eine interessante Möglichkeit zur Prävention von weiteren Finanzkrisen in Asien. Als regionaler Fonds könnte er auch nicht-regionale Länder mit politischen oder ökonomischen Interessen in der Region teilnehmen lassen.

Die Kooperation, die hier mit Japan einsetzen könnte, liegt in einem gemeinsamen Eintreten für den AMF. Wie sich aus Äußerungen des japanischen Finanzministers erkennen läßt, ist Japan weiterhin an der Einführung eines AMF interessiert. Sein Scheitern 1997 am amerikanischen Widerstand zeigt jedoch, daß westliche Fürsprecher vonnöten sind, um Befürchtungen vor einer „Verselbständigung“ Asiens zu zerstreuen. Deutschlands Beitrag bei dieser Art von Kooperation würde also mehr, als in der Bereitstellung von finanziellen Mitteln, darin liegen, den AMF international zu vertreten. Auch hier kann Deutschland wieder als Garant für eine globale Entwicklungspolitik auftreten und so dem AMF die vor allem benötigte amerikanische Unterstützung verschaffen.

Ähnlich wie bei der als erstes diskutierten Kooperationsmöglichkeit sind auch hier die Chancen auf eine Verbesserung der wirtschaftlichen Kontakte in die asiatische Region gegeben, und daher ist nicht nur im Hinblick auf eine Verbesserung der Situation in Asien, sondern auch für eine eigene Interessenverfolgung ein solches Engagement für Deutschland attraktiv.

Durch eine Kooperation mit Japan kann also innerhalb eines multilateralen Rahmens auf finanzieller Ebene ein sinnvoller Beitrag zur Krisenbewältigung geleistet werden und mehr für die Position Deutschlands in Asien bewirkt werden als durch bloße Zahlungen in den IMF. Im folgenden soll auf die Kooperationsmöglichkeiten auf (finanz-)technischer Ebene eingegangen werden.

\section{Möglichkeiten der Kooperation im Bereich technischer bzw. finanztechnischer Zusammenarbeit}

Für eine mögliche Kooperation Deutschlands und Japans auf der Ebene der Prävention von Finanzkrisen könnte der technischen bzw. der finanztechnischen Zusammenarbeit eine besondere Rolle zukommen. Anders als bei der FZ wären hier gemeinsame Projekte mit einem weitaus geringeren Kapitalbedarf möglich.

Wie bereits erwähnt, wird das unterentwickelte Bankensystem der asiatischen Entwicklungsländer als ein, oder vielleicht sogar als der Hauptgrund für die asiatische Finanzkrise gesehen. Daher ist es notwendig, über eine Neustrukturierung des asiatischen Bankensystems zu diskutieren, auch um zukünftig die Gefahr des moral hazard zu verringern. In diesem Zusammenhang sehen wir eine sinnvolle Möglichkeit der Zusammenarbeit Deutschlands und Japans: das Einrichten von gemeinsamen Beraterteams, die in den betroffenen Ländern Know-how für die notwendigen Reformen des Bankensystems vermitteln. Deutschland hat durch eine lange Tradition im Bereich der Bankenaufsicht, geprägt durch sein Kreditwesengesetz, gerade hier sehr viel Expertise aufzuweisen. Eine entscheidende Frage ist jedoch, warum Deutschland in diesem Bereich ausgerechnet mit Japan zusammenarbeiten sollte. Denn, genauso wie das asiatische Bankensystem, wird auch das japanische Modell international stark kritisiert. So summierte sich der Anteil der Problemkredite der 15 größten japanischen Kreditinstitute zum Ende des Geschäftsjahres am 31. März

9 Bilaterale systematische Überwachungstätigkeit des IMF der Währungspolitik seiner Mitgliedsländer, die - in der Regel einmal jährlich - in Form von Konsultationen durchgeführt wird. Dabei sammelt ein IMF-Stab wirtschaftliche und finanzielle Informationen und erörtert mit den Behörden die wirtschaftliche Entwicklung sowie deren Geld-, Fiskal- und Strukturpolitik. 
1999 auf mehr als 20 Billionen Yen (rund 30 Milliarden DM). Im Gegensatz zu Deutschland verfügt Japan jedoch über die nötigen Kontakte zu der Region. Auch das durch die IMF-Interventionen hervorgerufene Mißtrauen in der Region gegenüber dem Westen würde es Deutschland schwer machen, allein eine entscheidende Rolle in der Region zu spielen. Japan ist für die asiatischen Entwicklungsländer nicht nur ein wichtiger Absatzmarkt und eine Importquelle für Zwischenprodukte, sondern übt auch eine Vorbildfunktion aus (Look East). So wäre es auch im Sinne der Entwicklungsländer Asiens, daß, wenn sie sich beraten lassen, dieses von jemandem geschieht, der sich auch in der Region, mit seiner Kultur und seinen Verhaltensweisen, auskennt. Außerdem war Japan das einzige Land, das den asiatischen Entwicklungsländern unabhängig von den internationalen Organisationen nach dem Ausbruch der Krise finanzielle Hilfe leistete und zeigte, daß es bereit ist, auch größere Summen zur Verfügung zu stellen

Kurz ausgedrückt läßt sich sagen, daß Deutschland die Expertise und Japan die Kontakte hat. Auch im eigenen Interesse wäre es für Deutschland als exportorientierte Nation vorteilhaft, wenn sich solch eine Krise nicht wiederholt, da diese nicht nur regionale, sondern auch globale Folgen hatte. Dies gilt ebenso für Japan. Und im Hinblick auf das eigene Bankensystem könnte eine Zusammenarbeit mit Deutschland für Japan aufgrund eventueller Lerneffekte Vorteile haben.

Zur Realisierung dieses Konzeptes bedarf es aber auch der Zustimmung der internationalen Organisationen. Der IMF, der eigentlich mit der Aufgabe der Prävention von Finanzkrisen betraut ist, könnte eventuell für einen derartigen Ansatz gewonnen werden, da er auch im Jahresbericht 1998 dafür plädiert, ,...daß die internationale Gemeinschaft größere Anstrengungen unternehmen müsse, um im Rahmen der Krisenprävention neu entstehende Anfälligkeiten zu identifizieren...". Es müßte jedoch deutlich gemacht werden, daß dieses Projekt weniger eine Einmischung in die Angelegenheiten des IMF bedeuten, sondern vielmehr eine Ergänzung darstellen würde. Aus der Tatsache heraus, daß der IMF den Entwicklungsländern Bedingungen bei der Kreditvergabe stellt, zu denen auch die Reformierung des Bankensystems zählt, könnte dieses Projekt sowohl dem IMF als auch den Entwicklungsländern helfen, die Forderungen schneller und effektiver zu erfüllen.

Obwohl eine solche Kooperation auch Aufgaben umfassen könnte, die im Aufgabenbereich des IMF liegen, würde es trotzdem nicht die Kompetenzen des IMF schmälern. Es hätte eher eine Erweiterung der Aufgaben des IMF zur Folge, da dieser, bedingt durch den Personalmangel, bisher nicht in der Lage war seine Kompetenzen auf andere Ebenen auszuweiten.

Eine weitere Zusammenarbeit wäre innerhalb von multilateralen Organisationen, wie IMF oder Weltbank, möglich. Innerhalb dieser Organisationen könnten Japan und Deutschland dafür eintreten, sich mehr für ein stabiles internationales Währungssystem einzusetzen. So wäre innerhalb des IMF die Gründung eines Gremiums denkbar, das sich verstärkt mit der Stabilisierung von Währungen beschäftigt. Es hätte die Aufgabe, sich nach Ausbruch einer Krise um die Stabilisierung der betroffenen Währungen zu kümmern. Dadurch könnte unter Umständen die Krise abgefedert werden, so daß mehr Zeit zur Verfügung steht, um sich den eigentlichen Ursachen der Finanzkrise zuzuwenden. Auch Japan hätte großes Interesse an einem solchen oder ähnlichen Projekt, wie Japans Ministerpräsident, Keizo Obuchi, bei seinem Besuch in Deutschland im Januar dieses Jahres ausdrücklich betonte. Eine Verhinderung der Währungsabwertungen in den asiatischen Entwicklungsländern wäre eine Politik, die Deutschland, als eine der größten Exportnationen der Welt, auch im eigenen Interesse verfolgen könnte, denn Abwertungen bedeuten ein größeres Konkurrentenaufkommen und ein Verlust von potentiellen Absatzmärkten aufgrund von gestiegenen Importpreisen.

Eine weitere Idee, die es sich lohnt zu diskutieren, ist die Gründung eines deutsch- japanischen Forums zur Erfassung von Daten und Statistiken bezüglich der Entwicklungsländer. Im Jahresbericht 1998 des IMF wurde eine bessere Erfassung von Daten gefordert, die noch keinen internationalen Standards unterliegen. Wobei auch gesagt wurde, daß bei denjenigen Ländern, die nicht in der Lage seien, selbständig diese Daten zu beschaffen, eine technische Hilfe seitens des Fonds im Rahmen seiner Möglichkeiten erforderlich sei. Da der IMF durch Personalmangel gekennzeichnet ist, könnte hier eine Zusammenarbeit zwischen Deutschland und Japan Sinn machen. Japan und Deutschland sind weltweit führend in der Datenerfassung und verarbeitung und hätten somit genügend Expertise, um die benötigten Daten erfassen zu können. Dieses 
Forum hätte dann die Aufgabe, sich in den jeweiligen Ländern diese Angaben und Daten, teils durch Umfragen, zu beschaffen, um sie danach auszuwerten. Die Auswertungen würden dann an den IMF und die Ländern selbst weitergeleitet werden. Der IMF hätte dadurch die Möglichkeit, dementsprechend die richtigen Maßnahmen zur Bewältigung der Krise einzuleiten. Deutschland und Japan sollten in ihre Aufgabenstellung auch die Ausbildung von Personal aus Entwicklungsländern einbeziehen.

Desweiteren ist als mögliche Zusammenarbeit zwischen den beiden Ländern die Gründung einer deutschjapanischen Akademie denkbar, die sich auf die Ausbildung von Finanzbeamten konzentriert. Die notwendigen Reformen und Verbesserungen in den Entwicklungsländern bedürfen einer kompetenten Verwaltung, die in der Lage ist, diese Reformen zu entwerfen und durchzuführen. Für die asiatischen Länder ist es wichtig, über Beamte mit dem nötigen Know-how verfügen zu können, welches in dieser Akademie vermittelt werden sollte. Von seiten Deutschlands könnten bei solch einem Projekt aufgrund seines Ausbildungssystem, das auch durch die Erwachsenenbildung geprägt wird, sicherlich interessante Anregungen erfolgen. Japans Vorteile lägen, wie schon genannt, in den guten Kontakten zur Region, sowie in der Bereitschaft mit enormen finanziellen Mitteln zur Entwicklung der asiatischen Entwicklungsländer beizutragen.

Als Fazit läßt sich festhalten, daß es durchaus Möglichkeiten der Zusammenarbeit zwischen Deutschland und Japan auf der Ebene der technischen bzw. der finanztechnischen Hilfe zur Prävention von Finanzkrisen gibt, und diese, auch im Hinblick auf das Eigeninteresse Deutschlands und Japans, einer Diskussion wert sind.

\section{Empfehlung zur Umsetzung entwicklungspolitischer Kooperationen}

Deutschland und Japan bieten Potential für verstärkte gemeinsame Kooperation auf dem Gebiet der Entwicklungszusammenarbeit. Diese Aussage wird durch die Ergebnisse der vorangegangenen Kapitel gestützt. Ihre mögliche Umsetzung soll nun deshalb an dieser Stelle skizziert werden, wobei auf die bisher in dieser Arbeit angesprochenen Konzepte und Möglichkeiten zurückgegriffen wird.

Deutschland sollte über entsprechende Regierungsstellen folgende Vorschläge im Bereich der „klassischen“ Entwicklungspolitik unterbreiten:

Länderstudien, -berichte und -konzepte sollten ausgetauscht und verglichen werden. Wo bereits heute engere Verbindungen bestehen, etwa auf der Botschaftsebene, könnten solche Einschätzungen womöglich sogar gemeinsam erarbeitet oder zumindest frühzeitig aufeinander abgestimmt werden. Dieses Vorgehen könnte zunächst in Form eines Modellprojektes an ausgewählten Botschaften durchgeführt werden, damit die Möglichkeit der Umsetzung überprüft werden kann. Dazu sollten wenigstens zwei Botschaften in zwei Entwicklungsländern bestimmt werden. Die Ergebnisse dieser Kooperation sollten dann miteinander diskutiert werden, um eine Fortführung oder aber auch eine Einstellung solcher Projekte zu beschließen. Die Kosten eines solchen Modellprojektes sind als eher gering einzustufen und könnten, bei der erkennbaren Chance zu Synergieeffekten, auch auf japanischer Seite auf Zustimmung stoßen. Im Vorfeld der Studienerstellung müssen die Kriterien der Beurteilung abgestimmt werden, wodurch beide Seiten auch zu einer Überprüfung ihrer Kriterien kommen und eventuelle Relativierungen vorgenommen werden können.

Es sollte der Versuch gemacht werden, deutsche und japanische NGOs einander näher zu bringen. Zwar existieren nationale und internationale Netzwerke von NGOs, aber gerade im Hinblick auf die japanische NGO-Szene ist eine stärkere Vernetzung notwendig. Denn werden auch japanische NGOs in Zukunft noch in weit stärkerem Maße in die Entwicklungspolitik mit einbezogen, dann ist möglichst frühe Vernetzung dringend notwendig, um bei der konkreten Arbeit in Entwicklungsländern voneinander und miteinander profitieren zu können. AA und BMZ könnten hierbei als Initiator in Erscheinung treten. Die Kontakte zu deutschen NGOs auf dem Gebiet der Entwicklungshilfe sollten genutzt und auf ein gemeinsames Vorgehen 
gegenüber Japan abgestimmt werden. Um Ausgrenzungen zu vermeiden, sollte die Kontaktaufnahme über den Dachverband der deutschen NGOs (VENRO) geschehen.

Intensivere Kooperation zwischen den deutschen und japanischen Durchführungsorganisationen GTZ und JICA ist anzuraten. Hierzu sollte der bereits aufgenommene Personalaustausch verstärkt werden. Gegenseitiger Erfahrungsaustausch und die Möglichkeit voneinander zu lernen, stehen im Vordergrund dieser Empfehlung. Falls der politische Wille zu gemeinsamen Projekten vorhanden ist, kann die praktische Durchführung nur sinnvoll funktionieren, wenn Arbeits- und Denkweisen auf einen gemeinsamen Nenner gebracht werden können. Nur so können Mehrkosten, die ja gerade nicht entstehen sollen, verhindert werden.

Gemeinsame Entwicklungsprojekte sollten einen ähnlichen Modellcharakter erhalten wie die angesprochene Studienkooperation. Die Empfehlung ist auch hierbei, mehr als ein Projekt zu initiieren, damit Vergleichsmöglichkeiten für die Evaluation entstehen. Um Ressentiments zu vermeiden, müssen mehrere Vorbedingungen geschaffen werden. Die Grundstruktur sollte paritätisch sein. Das schließt nicht aus, daß einer der Partner mehr Mittel oder mehr Personal zur Verfügung stellt, weil ja gerade aus diesen Verschiedenheiten die gewünschten Synergieeffekte entstehen sollen. Dennoch ist eine Gleichstellung bei Grundsatzentscheidungen notwendig, da sonst die Kooperationsbereitschaft in Frage gestellt werden muß. Das Land, in dem Entwicklungshilfe geleistet werden soll, muß stark in die Kooperation eingebunden werden. Die möglichen Vorteile müssen klar dargelegt werden, um eine negative Haltung nicht aufkommen zu lassen. Die Transparenz der Entscheidungs- und Durchführungsprozesse muß gewährleistet sein, da das Entwicklungsland die schwächste Verhandlungsposition innehat. Die Seriosität der beiden starken Partner muß betont werden, damit nicht von vornherein ein Gefühl der Unterlegenheit auf der Seite des Entwicklungslandes die Kooperation behindert oder scheitern läßt. Projekte sollten deshalb stark den Charakter der Krisenursachenbekämpfung tragen, damit nicht der Verdacht der ökonomischen Exploitation entsteht.

Die Möglichkeit zur Kooperation mit Japan auf dem Gebiet der Krisenreaktion in Form von PKOs ist als eher gering einzustufen. Nur bei der Nothilfe lassen sich Anknüpfungspunkte vertreten, wobei allerdings Nothilfemaßnahmen in der Regel besser über bestehende internationale oder multilaterale Organisationen abgewickelt werden. Die Organisationsform sollte durch den Charakter die bereits genannten ,ad-hoc Koalition" gekennzeichnet sein.

Wenn Erfolge in ad-hoc Koalitionen oder informellen Gesprächen feststellbar sind, dann könnte in einem weiteren Schritt eine gemeinsame Institution geschaffen werden, die eine engere und genauere Koordination zum Ziel haben müßte. Auf dem Weg dahin wäre es denkbar, auf beiden Seiten Koordinatoren zu benennen, die in den jeweiligen Ministerien und Organisationen verantwortlich für gegenseitigen Austausch sind. Ergeben diese formalisierten Vorgehensweisen meßbare Ergebnisse, dann kann ein solches Gremium dauerhaft eingerichtet werden.

Deutschland sollte es vermeiden, gegenüber Japan allzu „besserwisserisch“ aufzutreten. Mißperzeptionen sind gerade zwischen Partnern unterschiedlicher Kulturkreise häufig der Grund für das Scheitern engerer Beziehungen. Diese Empfehlungen zielen aber gerade auch darauf ab, die deutsch-japanischen Beziehungen zu verbessern. Vielmehr sollte Japan eine gemeinsame Kooperation durch die aufgezeigten Vorteile nahe gebracht werden.

Neben der „klassischen“ Entwicklungspolitik bieten sich aber auch im Bereich der Krisenprävention/ reaktion finanzieller Krisen interessante Anknüpfungspunkte für eine Kooperation Deutschlands und Japans an. Ein Engagement in diesem Bereich ermöglicht eine Interessensverfolgung, die über ein reines entwicklungspolitisches Interesse hinausgeht.

Deutschland kann durch einen aktiven, direkten Beitrag zur Krisenbewältigung, anstatt durch bloße Zahlungen an den IMF, einen größeren Einfluß in der Region Asien gewinnen. Desweiteren kann gerade in der Kooperation mit Japan eine sinnvolle Hilfe zur Krisenbewältigung geleistet werden. Denn vor allem aufgrund der Synergieeffekte, die in der Region Asien gebildet werden können, ist eine Zusammenarbeit aussichtsreich. Japan mit seiner Bereitschaft, umfangreiche Geldmittel zur Verfügung zu stellen und mit 
seiner innerhalb Asiens anerkannten Führungsposition auf der einen Seite und Deutschland auf der anderen Seite, mit seiner Expertise und unbefangeren Haltung gegenüber den USA, aus der heraus es, falls notwendig, eine Mittlerposition zwischen Japan und USA einnehmen könnte.

Folgende Empfehlungen sollten für eine Kooperation mit Japan in Betracht gezogen werden:

Um deutlich zu signalisieren, daß bei einem gemeinsamen Engagement, das zur Bewältigung der Asienkrise beiträgt, keine eigenständige Politik betrieben werden soll, ist die Aufstellung eines Memorandums sinnvoll: beide Länder bekennen sich in diesem öffentlich dazu, die technische und finanzielle Hilfe im Rahmen der vom IMF geforderten Strukturanpassungen durchzuführen und zeigen damit, daß sie keine Politik gegen die USA beabsichtigen, sondern sich vielmehr in den globalen Kontext einfügen wollen.

Was konkret die Umsetzung der im vorangegangenen Kapitel vorgestellten Möglichkeiten betrifft, so müßte eine Zusammenarbeit mit Japan innerhalb der ADB, respektive Fondsgründung, initiiert werden durch das BMZ, da es federführend für die Regionalbanken ist. Ansprechpartner auf der japanischen Seite ist das MoF. Bei einer Unterstützung des AMF von deutscher Seite wäre eine Einbeziehung des Wirtschaftsministeriums sinnvoll, da dieses aufgrund seiner internationalen Bedeutung für die Anerkennung eines AMF von amerikanischer Seite sorgen könnte.

In bezug auf die technische Unterstützung, die von Deutschland und Japan vor allem im Bereich der Beratung zu strukturellen Reformen im Bankensektor geleistet werden könnte, müßte eine Initiierung durch das BMZ oder auch das Wirtschaftsministerium erfolgen.

Was die Durchführung der Beratung anbetrifft, so ist eine Zusammenarbeit auf institutioneller Ebene, wie z.B. zwischen GTZ und JICA denkbar. Hier kann auf den bereits bestehenden Beziehungen aufgebaut werden. Es ist allerdings anzumerken, daß die GTZ solche Beratungsdienste bisher noch nicht durchführte. Aufgrund des Know-hows, das bei diesen Beratungen vermittelt werden soll, ist auch eine Einbeziehung der KfW sinnvoll, die in dieser Thematik Expertise aufweisen kann. Eine Zusammenarbeit mit der JDB ist denkbar, da auch hier bereits Kooperationserfahrung existiert. Hier ist allerdings auf die Problematik hinzuweisen, die bei der KfW in bezug auf technische Unterstützung besteht: diese darf von der KfW nur im Rahmen von finanzieller Zusammenarbeit geleistet werden. Das heißt, der investive Charakter muß überwiegen. Es soll an dieser Stelle die Frage in den Raum gestellt werden, inwieweit eine solche Beschränkung sinnvoll ist, oder aber eher Unterstützung verhindert.

Die getroffenen Empfehlungen lassen als Fazit den Schluß zu, daß Deutschland und Japan zukünftig in stärkerem Maße gemeinsam Entwicklungspolitik betreiben sollten. Dabei kann der Rahmen der traditionellen Entwicklungszusammenarbeit verlassen bzw. erweitert werden, um den Anforderungen heutiger Entwicklungsnotwendigkeiten gerecht zu werden. Die nach wie vor bestehenden Unterschiede in der Politiksetzung und -umsetzung werden geringer und könnten für eine gemeinsame und globale Entwicklungs- und Strukturpolitik genutzt werden. 


\section{Literaturverzeichnis}

ADB (Hg.) (1999): Address to the Board of Governors by President Tadao Chino, 30.04.1999, in: Internet: http://www.adb.org/AnnualMeeting99/chino.asp, Zugriff am 22.05.1999.

ANDERSEN, UWE (1995): Entwicklungspolitik / -hilfe, in: Woyke, Wichard (Hg.): Handwörterbuch Internationale Politik, 6. Auflage, Opladen 1995, S. 83-92.

ARASE, DAVID (1995): Buying power. The Political Economy of Japan's Foreign Aid, Boulder/London 1995.

BEHRENDT, JENS RAINER (1992): Die asiatische Entwicklungsbank, Hamburg 1992.

BMZ (1997): Grundlagen der deutschen Entwicklungszusammenarbeit, Materialien Heft Nr. 97, Bonn 1997.

BMZ (1998): Deutsche Entwicklungspolititk, Materialien Heft Nr. 98, Bonn 1998.

BMZ (1998): Jahresbericht 1997, Bonn 1998.

BUNDESREGIERUNG (1998): Pressemitteilungen, in: Internet: http://www.bundesregierung.de/pressemitteilungen, Zugriff am: 26.05.1999.

CORSETTI, G., P. PESENTI UND N. ROUBINI (1998): What caused the Asian Currency and Financial Crisis?, Rom 1998.

DIETER, HERIBERT (1998): Die Asienkrise und der IWF: Ist die Politik des internationalen Währungsfonds gescheitert?, INEF-Report Heft 29, 1998.

ECONOMIC COOPERATION BUREAU (Hg.) (1999): A Guide to Japan's Aid, in: Internet: http://www.mofa.go.jp/policy/oda/guide/index.html, Zugriff am: 21.04.1999.

EHRKE, MICHAEL (1996): Die japanische Entwicklungspolitik, in: Jahrbuch Dritte Welt, Beck, München 1996, S. 34-52.

ENSIGN, MARGIE M. (1992): Doing Good or Doing Well? Japans Foreign Aid Program, New York 1992.

FOERSTER, ANDREAS (1994): Japans Zusammenarbeit mit der Dritten Welt zwischen Entwicklungsorientierung und außenwirtschaftlichen Prioritäten, Berlin 1994.

FUJISAKI, TOMOKO, FORREST BRISCOE (1996): Japan as top donor, in: Pacific Affairs 4/1996, S. 519-539.

GOYDKE, TIM (1999): Die Auswirkungen der Asienkrise auf die entwicklungstheoretische Debatte in Asien, unveröffentlicht, 1999.

HOLTHUS, MANFRED, DIETRICH KEBSCHULL (1985): Beeinflussung der außenwirtschaftlichen Beziehungen der Bundesrepublik Deutschland durch die Entwicklungspolitik anderer OECDLänder, in: Holthus, Manfred, Dietrich Kebschull (Hg.): Die Entwicklungspolitik wichtiger OECDLänder, Band 1, Hamburg 1985, S. 51-69.

INTERNATIONALER WÄHRUNGSFONDS (1998): Der IWF im Geschäftsjahr 1997/98 S.25-54, in: Internet: http://www.imf.org/, Zugriff am: 04.06.1999.

JICA (1998): Annual Report FY 1997, in: Internet: http://www.jica.go.jp/Einfo/A report97/MAp040.html, Zugriff am: 21.04.1999.

KOPPEL, BRUCE M., ROBERT M. ORR (Hg.) (1993): Japan's Foreign Aid, Boulder, London 1993.

LERCH, HUBERT (1984): Die japanische bilaterale Entwicklungspolitik am Beispiel Afrikas, München 
1984.

MAY, BERNHARD (1989): Japans neue Entwicklungspolitik, München 1989.

MAY, BERNHARD (1994): Entwicklungshilfepolitik, in: Mayer, Hans Jürgen, Manfred Pohl (Hg.): Länderbericht Japan, Bonn 1994, S. 166-178.

MITI (Hg.) (1999): Asian Economic Crisis and Japan's Contribution, in Internet: http://www.miti.go.jp, Zugriff am: 22.05.1999.

MoFA (1998): ODA Summary 1997, in Internet: http://www.mofa.go.jp/policy/summary/1997/index.html, Zugriff am: 18.05.1999

MoFA (1999a): Japan‘s Official Development Assistance. Annual Report 1998, Tokyo 1999.

MoFA (1999b): Japan‘s Official Development Assistance. Summary 1998, Tokyo 1999.

NUSCHELER, FRANZ (1994): Japan als „aid leader“: Neue Entwicklungen in der japanischen Entwicklungspolitik, in: Pohl, Manfred: Japan 1993/94, Hamburg 1994, S. 163-180.

NUSCHELER, FRANZ (1995): Lern- und Arbeitsbuch Entwicklungspolitik, 4. Auflage, Bonn 1995.

NUSCHELER, FRANZ (1998): Die japanische Entwicklungspolitik in der Klemme der Wirtschafts- und Haushaltskrise, bisher unveröffentlichter Artikel, Kurzfassung in: Entwicklungspolitik, 17/18, 1998, S. 23-27.

NUSCHLER, FRANZ (1997): Entwicklungspolitik und Menschenrechte. Theorie und Praxis der Politischen Konditionalität in der japanischen Entwicklungspolitik; INEF-Report 24, 1997.

o.V. (1998): Die Koalitionsvereinbarung. Auszug aus entwicklungsrelevanten Passagen, in: E+Z Entwicklung und Zusammenarbeit, 12, 1998, S. 326-327.

o.V. (1999): ADB sagt Armut in Asien den Kampf an, in: Handelsblatt, 3.5.1999, S.10.

o.V. (1999): Entwicklungspolitik als Friedenspolitik. Interview mit Ministerin Heidemarie Wieczorek-Zeul, in: E+Z - Entwicklung und Zusammenarbeit, 1, 1999, S. 8-10.

OECD/DAC (1996): Devolopment Co-operation Review Series Japan; 1996 No.13, Paris 1996.

OECD/DAC (1998): Devolopment Co-operation Review Series Germany; 1998 No.29, Paris 1998.

POHL, MANFRED (1996): Deutschland und Japan - von wohlwollender Nichtbeachtung zum intensiven Dialog, in: Kaiser, Karl, Krause Joachim (Hg.): Deutschlands neue Außenpolitik, Bd. 3, München 1996, S. 155-160

RAGNITZ, JOACHIM (1996): Deutschland und die Gestaltung der Weltwirtschaft, in: Kaiser, Karl; Krause Joachim (Hg.): Deutschlands neue Außenpolitik, Bd. 3, München 1996, S. 63-76.

ROHDE, MIRIAM (1996): Japans staatliche Entwicklungshilfe (ODA): Ansätze zu einer neuen Entwicklungspolitik, in: Japan. Wirtschaft, Politik, Gesellschaft, Jg. 4/1996; S. 416-430.

SACHS, JEFFREY (1997): IMF is a power unto itself, 11.12.1997, in Internet: http://equity.stern.nyu.edu/ nroubini/asia/AsiaHomepage.html, Zugriff am: 21.05.1999.

SCHMUCK, OTTO (1995): Entwicklungspolitik, in: Weidenfeld, Werner; Wessels, Wolfgang (Hg.): Europa von A-Z, Bonn 1995, S. 122-125.

WELTBANK (Hg.) (1999): The World Bank Annual Report 1998, in Internet: http://www.worldbank.org, Zugriff am: 21.05.1999.

WESTERWELLE, ANGELIKA (1995): Staat und Wirtschaft in Japans bilateraler Entwicklungszusammenarbeit, Dissertation, Aachen 1995. 